\title{
Bargaining one-dimensional social choices *
}

\author{
Daniel Cardona ${ }^{\dagger}$ \\ Universitat de les Illes Balears \\ Clara Ponsatí \\ Institut d'Anàlisi Econòmica -C.S.I.C., Barcelona \\ Accepted, December 2006 \\ Running title: Bargaining social choices
}

\footnotetext{
${ }^{*}$ We are indebted to an anonymous referee for detailed comments, and to Ramon Faulí and Xavier Raurich for discussions. Financial support from the European Comission (CIT-2-CT-2004-506084), Ministerio de Educación y Ciencia (SEJ2006-02079, SEJ2006-05441, and CONSOLIDER-INGENIO 2010-CSD200600016) and CREA-Barcelona Economics is gratefully acknowledged.

${ }^{\dagger}$ Corresponding author: Dept. Economia Aplicada Universitat de les Illes Balears, Ctra. Valldemossa km. 7.5, 07071 Palma de Mallorca, Spain, e-mail:d.cardona@uib.es, Ph: (34) 971-172845 Fax: (34) $971-172389$
} 


\begin{abstract}
We analyze bargaining over the one-dimension characteristic of a public good among $n$ impatient players when decisions require $q$ favorable votes, $q \geq 2$. Stationary subgame perfect equilibrium strategies are characterized for all games with deterministic protocol. We provide a monotonicity condition (satisfied by all singlepeak, strictly quasi-concave and concave utilities) that assures uniqueness for every $q$ whenever player's utilities are symmetric around the peak. Without symmetry, the monotonicity condition assures uniqueness for qualified majorities, $q>n / 2$, provided that agents are sufficiently patient and utilities satisfy an additional regularity condition. Asymptotic uniqueness is assured for qualified majorities by imposing only the monotonicity condition.

Keywords: Bargaining, Voting, Qualified Majority, One-dimensional Policies, SinglePeaked Preferences, Public Good Location.

JEL classification numbers: C78, D71,D72, D74.
\end{abstract}




\section{Introduction}

This paper examines non-cooperative bargaining games in a classical environment of social choice: A group of individuals must collectively choose a public good in a one-dimensional interval of alternatives, over which individuals have single-peaked preferences. Examples are the location of a public facility, the adoption of a public policy, or the allocation of a budget among two public services.

It is well known ${ }^{1}$ that in these scenarios simple majority voting has very appealing properties: It selects a Condorcet winner (the peak of a median voter), an outcome that is immune to strategic manipulation and lies in the core of the underlying cooperative game.

Still, simple majority is sometimes indecisive ${ }^{2}$ and, more importantly, collective decisions often require levels of consensus other than a simple majority. For these situations, predictions must rely on detailed examination of the bargaining processes by which alternatives gather decisive support. In this paper we are concerned with such environments. We ask three main questions: What are collective decisions like when consensus over an alternative is bargained over time? Under what conditions is it possible to give a sharp prediction for the collective decision? How would this prediction depend on the intensity of the consensus required? To address these questions, we analyze games taking place over discrete time where a collective decision must be reached by a deterministic protocol

\footnotetext{
${ }^{1}$ See Moulin [9] and [10].

${ }^{2}$ With an even number of voters, the (weak) Condorcet winner is not unique.
} 
of alternating proposals and voting, under the assumption that agents are impatient. We consider voting procedures that require the approval of a quota of players ranging from minimal consensus to unanimity.

Our main message is that, for a wide range of utilities, the assumption that agents discount future utilities exponentially has the usual drastic effect in selecting a unique stationary equilibrium outcome. We show that, in spite of the public nature of the collective decision and the tight constraint of one-dimensionality, the bargaining problem is not radically different from negotiations to split a private surplus. While any efficient decision can be sustained as a stationary equilibrium among perfectly patient players, impatience plays a powerful role in selecting a single alternative. When the utilities are symmetric around the peak and satisfy a natural condition of monotonicity (satisfied by all continuous, strictly quasi-concave, and concave utilities) a unique stationary equilibrium is assured for every quota, $q=2$ to $n$, and for all discount factors. Without symmetry, the monotonicity condition assures the selection of a unique outcome only when collective decisions demand a (super)majority and agents are rather patient. We establish that, for all $q>n / 2$, monotonicity and an additional regularity condition assure a unique equilibrium provided that the agents are sufficiently patient. When the regularity condition is not imposed, monotonicity assures asymptotic uniqueness: as players become arbitrarily patient, all equilibrium outcomes converge to a unique limit (independently of the order of play).

The arguments underlying our results are distinct from those that apply in multilateral 
negotiations to share a pie under the unanimity rule. In a standard pie-sharing bargaining game where three or more players must reach a unanimous agreement to split a private surplus, impatience (combined with stationarity) has a dramatic effect and shrinks the set of equilibrium outcomes to a unique division of the surplus because: (a) to get acceptance the proposer must offer to each opponent a share that is worth exactly their present value of rejecting, and (b) since all shares must add up to one, each player's value of rejecting (assumed stationary) is determined by the shares that she must offer to receive approval when she is the proposer. The constraints implied by (a) and (b) are tight and determine equilibrium shares uniquely. ${ }^{3}$ This argument does not work in our set up because proposals cannot be customized to each voter: when the support of more than two players is needed, equilibrium proposals will easily deliver payoffs above continuation values to some responders.

Our focus also departs from arguments based on the link between the core and the set of stationary subgame perfect equilibria. When the players are perfectly patient, any allocation in the core can be sustained as a stationary subgame perfect equilibrium outcome (see Perry and Reny [12]). With an odd number of agents the core of a simple majority game contains a single alternative, the peak of the median voter. Consequently, under perfect patience, the unique stationary subgame perfect equilibrium outcome of bargaining games where decisions are taken by simple majority is the peak of the median voter. Since the stationary subgame perfect equilibrium correspondence is upper-

\footnotetext{
${ }^{3}$ Under qualified majority rules, things are more complicated because only the least demanding players need to be offered their expected continuation payoffs (see Eraslan [4]).
} 
hemicontinuous, games where players are sufficiently patient have a unique stationary subgame perfect equilibrium as well, with outcomes that remain close to the peak of the median voter. ${ }^{4}$ However, when a collective decision requires more than a simple majority, the underlying cooperative game has a large core and arguments based on core equivalence and upper-hemicontinuity do not help to attain uniqueness.

This paper is closely related to Banks and Duggan [1] and Cho and Duggan [3]. The social choices that we discuss are a particular case of the more general problem addressed by Banks and Duggan [1]. They consider a model of sequential bargaining where proposals, social alternatives, lie in arbitrary convex subsets of a multidimensional Euclidean space. Proposers are selected at random and agreement is reached by an arbitrary voting rule. They prove existence of equilibria under very general conditions, and they establish sufficient conditions for core equivalence. ${ }^{5}$ Their results, however, do not provide an explicit characterization of equilibria nor a discussion of conditions for uniqueness. The issue of uniqueness for one-dimensional problems is addressed in Cho and Duggan [3]. They show that under quadratic utilities, a game with random proposers and strong and proper decisive coalitions has a unique stationary subgame perfect equilibrium. ${ }^{6}$ Their result is based on the existence of a unique core player, a property that fails at quotas other than the simple majority. Our contribution is complementary; we establish (asymptotic) uniqueness for a wide range of preferences and consensus requirements, but our results

\footnotetext{
${ }^{4}$ See Jackson and Moselle [6].

${ }^{5}$ For set-ups like ours, where alternatives are in an interval, they show that equilibria (in pure strategies) exist; and that they are equivalent to core outcomes for perfectly patient players.

${ }^{6}$ When decisive coalitions are determined by a quota, only the simple majority is strong and proper.
} 
apply only to deterministic protocols. The issue of uniqueness for qualified majorities under random proposers remains open.

Jackson and Moselle [6] is also related. They consider alternatives that have two components, the location of a public good in an interval and the division of a private good among the agents. Their concern is the nature of coalitions that may form when decisions are taken with a procedure of alternating proposals and simple majority.

More indirectly, our work is also related to the literature that explores the (possible) equivalence between the equilibria of non-cooperative games and the core of underlying games in coalitional form (see Selten [14], Perry and Reny [12], Moldovanu and Winter [8] and Evans [5]). The equivalence results of all these models rely fundamentally on the assumption that players are perfectly patient; and might have the additional drawback of little content when the core is either empty or a large set. Chatterjee et al. [2] and Hart and Mas-Colell [7] present models where relaxing the assumption of perfect patience is instrumental in attaining sharper predictions within a large core. ${ }^{7}$ Neither of these two models, however, admits a formulation where the collective choice is the characteristic of a public good on an interval. ${ }^{8}$

\footnotetext{
${ }^{7}$ In Chatterjee et al. [2] any efficient stationary subgame perfect equilibrium converges (as the impatience diminishes) to the egalitarian allocation. In Hart and Mas-Colell [7] the stationary equilibrium outcomes converge to a "Mashler-Owen consistent value payoff configuration" (which are unique and lie in the core for specific formulations of the characteritic function).

${ }^{8}$ In our set up utilities are not transferable, and coalitional payoffs usually do not satisfy superadditivity, convexity or comprehensiveness. Chatterjee et al. [2] assume that the underlying game in coalitional form has transferable utility and is superadditive. In Hart and Mas-Colell [7] coalitions can attain payoffs
} 
In the next section, the general bargaining model is presented. In section 3 we characterize stationary subgame perfect equilibria and establish conditions assuring existence. Conditions for uniqueness of the equilibrium are explored in section 4 . The proofs omitted in the main text are in the Appendix.

\section{The Model}

A set of agents $I=\{1,2, \ldots, n\}$ must collectively choose an alternative $x$ in the interval $[0,1]$. The instantaneous preferences of each $i \in I$ are described by a continuous and strictly quasi-concave utility function, $u_{i}:[0,1] \rightarrow \Re_{+}$. Note that strict quasi-concavity and continuity imply single-peakedness and strict monotonicity; that is, there is a unique $p_{i} \in[0,1]$ such that $p_{i} \in \arg \max \left\{u_{i}(x): x \in[0,1]\right\}, u_{i}(x)<u_{i}(y)$ for $x<y \leq p_{i}$, and $u_{i}(x)>u_{i}(y)$ for $p_{i} \leq x<y$. The permutation of the peaks profile $\left(p_{1}, \ldots, p_{n}\right)$ in increasing

order is denoted by $\left(p^{1}, \ldots, p^{n}\right)$ with $p^{k}$ denoting its $k$-th term. To avoid trivial scenarios we assume that at least two players have different peaks, and we set $p^{1}=0$ and $p^{n}=1$ without loss of generality.

Some interpretations of this social choice problem are the following:

1. Choosing a policy or appointing a public official when the ideological contest concerns a single issue.

2. Selecting the location of a public facility between two cities connected only by one that lie in sets that are convex and comprehensive. 
road.

3. Deciding on the level of production of a public good subject to non-decreasing marginal costs, under equal sharing of the costs. Strictly convex preferences over public good and wealth combinations are represented by a single-peaked utilities over the public good space.

4. Allocating a fixed budget to finance the production of two public goods. If the preferences of the players are quasi-concave and increasing with respect to both goods, the two-dimension problem can be reduced to the present one-dimensional decision problem.

Decisions are bargained over discrete time $t=0,1, \ldots$ with an infinite horizon. The timing of the game is as follows. At $t=0$ player 1 selects a proposal $x \in[0,1]$ and sequentially the rest of players either accept or reject it. If $x$ is accepted by at least $q-1$ responders, $2 \leq q \leq n$, the collective decision $x$ is implemented and the game ends. Otherwise, the game moves to period $t=1$ where 2 becomes the proposer. And so on. The process continues until a proposal is supported by at least $q$ players (one of them the proposer). The utility of perpetual disagreement - where agents forego consumption of the public good - is normalized to zero so that players unanimously (weakly) prefer any agreement to perpetual disagreement. Upon termination of the game at $t$ with decision $x$ the payoff of a player $i$ is given by $\delta^{t} u_{i}(x)$ where $\delta \in(0,1)$ denotes the common ${ }^{9}$ discount factor.

\footnotetext{
${ }^{9}$ This is for convenience, the results go through with player-specific discount factors.
} 
Remark. For $n=2$, although the set of feasible agreements is not necessarily convex in the utility space, the preceding model is strategically equivalent to the standard bargaining game of Rubinstein [13]. Things change for $n \geq 3$ because the standard requirements over utility sets easily break down. Consider, for instance, the example displayed in Figure 1 where the utility set is neither convex nor comprehensive.

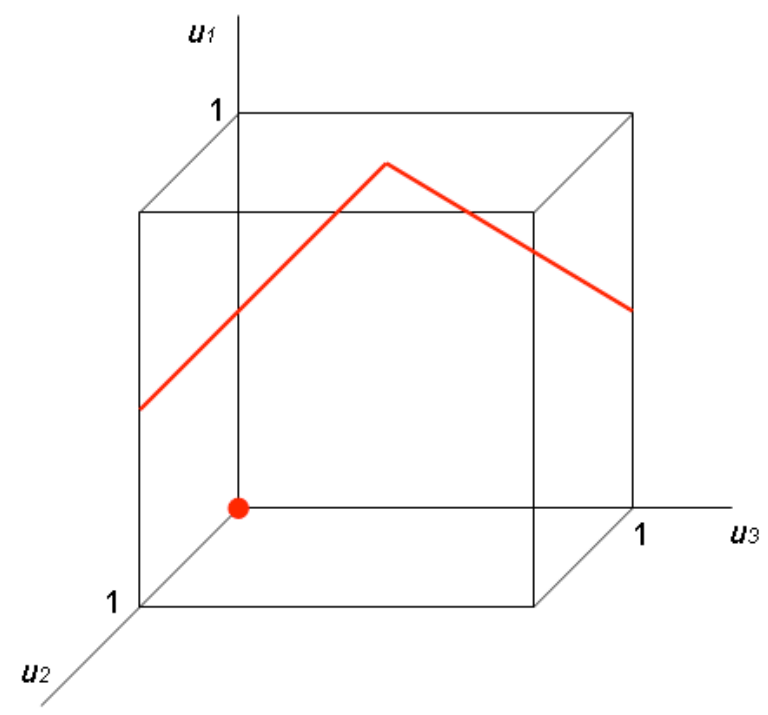

Figure 1. Feasible utilities set for $u_{1}(x)=1-|x-1 / 2|, u_{2}(x)=1-x$ and $u_{3}(x)=x$.

Histories, strategies and subgame perfect equilibria are defined in the standard way. We focus our attention to stationary subgame perfect equilibria (henceforth SSPE). At any SSPE agents play history independent strategies that constitute a subgame perfect equilibrium. Thus, whenever they act as proposers, agents select the same alternative; and they always use the same decision rule to accept/reject the proposals of a given 
opponent.

\section{Characterization and Existence}

In what follows, we fix $\delta \in(0,1)$ and a profile of utility functions $\left(u_{1}, \ldots, u_{n}\right)$ and characterize SSPE for the full range of quotas $q=2$ to $n$. The stationary nature of strategies crucially simplifies the characterization. First, the actions of all players depend only on the current state (who is the proposer, what is the proposal), so that expected payoffs at any subgame of a given equilibrium are fixed and independent of the history of play. Second, disagreement cannot prevail in equilibrium because it offers the least payoff to every player. Furthermore, for any strategy profile leading to an agreement with some delay, there is a profitable deviation that anticipates agreement. Therefore the following holds:

Lemma 1 Immediate Agreement. In any SSPE agreement is immediate.

An SSPE is characterized by a vector of proposals and acceptance rules, one for each player, that are mutually best responses. Consider a proposer $i$ that anticipates an immediate decision on alternative $x \in[0,1]$ if the negotiation enters into the next period. What alternative must she propose to attain the best possible agreement in the current period? She must propose an alternative $y$ that is acceptable, $u_{j}(y) \geq \delta u_{j}(x)$, for at least $q-1$ opponents $j$ in $I$. To assess what alternatives can receive consensus to defeat the prospect of agreement at $x$ tomorrow (and to compute which one is best) we must, first, 
account for the fact that utilities increase up to the peak and then decrease; and second, we need to keep track of what responders find each proposal acceptable. Although there is no fundamental obstacle, that requires the bit of notation that we introduce next.

For each utility function $u$ with peak at $p$, we define its associate left and right functions as $l:[0, p] \rightarrow \Re$ and $r:[p, 1] \rightarrow \Re$, where $l(x)=u(x), r(x)=u(x)$ (the notation $l_{i}$ and $r_{i}$ will be used if necessary). The trade-offs between alternatives and delay for player $i$ are now easily evaluated: an immediate agreement at an alternative $y$ dominates the prospect of agreement at $x$ with one period of delay provided that $u_{i}(y) \geq \delta u_{i}(x)$; that is, if and only if $y \in\left[\underline{\alpha}_{i}(x), \bar{\alpha}_{i}(x)\right]$, where

$$
\begin{aligned}
& \underline{\alpha}_{i}(x)= \begin{cases}l_{i}^{-1}\left(\delta u_{i}(x)\right) & \text { if } \delta u_{i}(x) \in\left[u_{i}(0), u_{i}\left(p_{i}\right)\right], \\
0 & \text { otherwise }\end{cases} \\
& \bar{\alpha}_{i}(x)= \begin{cases}r_{i}^{-1}\left(\delta u_{i}(x)\right) & \text { if } \delta u_{i}(x) \in\left[u_{i}(1), u_{i}\left(p_{i}\right)\right], \\
1 & \text { otherwise. }\end{cases}
\end{aligned}
$$

When the approval of only $m$ players in a set $P \subseteq I$ is needed for the success of a proposal, not all acceptance thresholds $\underline{\alpha}_{i}(x)$ and $\bar{\alpha}_{i}(x)$ are relevant. To keep track of which ones are we use the following notation: rank the elements of $\left\{\underline{\alpha}_{j}(x): j \in P\right\}$ and $\left\{\bar{\alpha}_{j}(x): j \in P\right\}$ in increasing and decreasing order respectively, and denote by $\underline{\alpha}^{P, m}(x)$ and $\bar{\alpha}^{P, m}(x)$ the $m$ - th terms in these rankings. Thus, when the approval of $m$ players $i$ in $P$ is necessary, proposal $z$ beats the prospect of decision $x$ in the next period if and only if $z \in\left[\underline{\alpha}^{P, m}(x), \bar{\alpha}^{P, m}(x)\right]$. 
Henceforth, whenever no confusion arises, we will write $\underline{\alpha}(x) \equiv \underline{\alpha}^{I, q}(x)$ and $\bar{\alpha}(x) \equiv$ $\bar{\alpha}^{I, q}(x)$. We are now ready to provide a rather simple characterization of the best responses.

Lemma 2 Best Responses. Consider the game under quota $q$. For each $x \in[0,1]$ the best response of proposer $i$ at $t$ when an immediate agreement $x$ prevails at $t+1$ is to propose $\chi_{i}(x)$, where $\chi_{i}:[0,1] \rightarrow \Re$ is defined as

$$
\chi_{i}(x)= \begin{cases}\bar{\alpha}^{I, q}(x) & \text { if } p_{i}>\bar{\alpha}^{I, q}(x), \\ \underline{\alpha}^{I, q}(x) & \text { if } p_{i}<\underline{\alpha}^{I, q}(x), \\ p_{i} & \text { otherwise. }\end{cases}
$$

Proof. A proposal $y$ by player $i$ is acceptable upon the prospect of agreement at $x$ with one period of delay provided that $y \in A_{j}(x)=\left[\underline{\alpha}_{j}(x), \bar{\alpha}_{j}(x)\right]$ for at least $q-1$ responders $j \neq i$; that is, $y \in A^{i, q}(x)=\left[\underline{\alpha}^{I \backslash\{i\}, q-1}(x), \bar{\alpha}^{I \backslash\{i\}, q-1}(x)\right]$. Since the $A_{j}(x)$ are connected and contain $x$ for all $j \in I$, it is immediate that $A_{i}(x) \cap A^{i, q}(x) \neq \emptyset$. Hence, for any proposer $i \in I$, there exists proposals that are acceptable at least to $q-1$ responders and that $i$ herself prefers over delay.

The best proposal of player $i$ in $A_{i}(x) \cap A^{i, q}(x)$ must maximize $u_{i}$ in $A^{i, q}(x)$. Furthermore, maximizing $u_{i}$ in $A^{i, q}(x)$ is equivalent to maximizing $u_{i}$ in $A^{q}(x)=\left[\underline{\alpha}^{I, q}(x), \bar{\alpha}^{I, q}(x)\right] \subseteq$ $A^{i, q}(x)$. To check this claim assume that the best alternative in $A^{i, q}(x)$, say $y$, is not the best alternative in $A^{q}(x)$; i.e. $y \in\left[\underline{\alpha}^{I \backslash\{i\}, q-1}(x), \underline{\alpha}^{I, q}(x)\right) \cup\left(\bar{\alpha}^{I, q}(x), \bar{\alpha}^{I \backslash\{i\}, q-1}(x)\right] \neq \emptyset$. If $y \in\left[\underline{\alpha}^{I \backslash\{i\}, q-1}(x), \underline{\alpha}^{I, q}(x)\right) \neq \emptyset$ then $p_{i} \leq y<\underline{\alpha}^{I, q}(x)$ since otherwise a proposal $y^{\prime}>y$ would be preferred by $i$ and accepted by at least $q-1$ players. Moreover, since $\underline{\alpha}_{i}(x) \leq p_{i}$ 
we must conclude that $y>\max \left\{\underline{\alpha}^{I \backslash\{i\}, q-1}(x), \underline{\alpha}_{i}(x)\right\} \geq \underline{\alpha}^{I, q}(x)$, which is a contradiction. The symmetric argument rules out $y \in\left(\bar{\alpha}^{I, q}(x), \bar{\alpha}^{I \backslash\{i\}, q-1}(x)\right] \neq \emptyset$. Hence, for all $i$, the optimal proposal when agreement on $x$ prevails next period in case of rejection is to propose an $y$ maximizing $u_{i}$ in $A^{q}(x)=\left[\underline{\alpha}^{I, q}(x), \bar{\alpha}^{I, q}(x)\right]=[\underline{\alpha}(x), \bar{\alpha}(x)]$. If $p_{i}$ lies in this interval then that is the best response proposal of player $i$; otherwise she must propose $\underline{\alpha}(x)$ or $\bar{\alpha}(x)$, whatever is closer to $p_{i}$.

We may now give a characterization of SSPE outcomes and establish that they exist.

Proposition 3 Characterization And Existence. Consider a game under quota q. The following statements hold:

(i) An alternative $x$ is an SSPE outcome if and only if

$$
x=\chi_{1}\left(\chi_{2}\left(\ldots \chi_{n-1}\left(\chi_{n}(x)\right)\right)\right),
$$

where, for all $i \in I, \chi_{i}(x)$ are defined by Eq. (1).

(ii) An equilibrium exists.

Proof. First observe that restricting attention to pure strategies is without loss of generality (see the Appendix for a detailed proof of this claim).

Consider an SSPE that yields an immediate decision $x$. By stationarity, this outcome prevails any time $t$ that player 1 proposes. Therefore, at period $t-1$ her predecessor in the protocol, $n$, must propose $\chi_{n}(x)$. Taking into account that at a stationary strategy player 1 proposes the same alternative $x$ whenever she intervenes as the proposer, (i) follows by recursive use of Eq. (1). 
It is immediate that the $\chi_{i}($.$) are continuous so that$

$$
w(x)=\chi_{1}\left(\chi_{2}\left(\ldots \chi_{n-1}\left(\chi_{n}(x)\right)\right)\right)
$$

is also a continuous. Moreover, by construction $0 \leq w(0) \leq 1$ and $0 \leq w(1) \leq 1$. Hence, the existence of a $x \in[0,1]$ satisfying $x=w(x)$ follows by Brouwer's fixed point theorem and the proof of (ii) is complete.

The existence of an SSPE is not a surprise (in fact, the existence result of Banks and Duggan [1], for the case of random proposers, is substantially more general); however, the present explicit characterization provides the tools to explore conditions for uniqueness.

\section{Uniqueness}

We now build on Proposition 3 to address conditions assuring uniqueness of the SSPE.

At this point it is useful to recall the classical argument for uniqueness of the equilibrium due to Rubinstein [13]: when two agents bargain to split a private surplus, a player expecting a large share is more ready to give up some gains to anticipate agreement than a player expecting a small share. Because equilibrium proposals must balance this trade-off for both players, and because what one agent gains the other must lose, uniqueness is assured provided that the surplus-delay trade off is strictly monotonic. In the present environment, the gain of an agent does not necessarily assure a loss to all her counterparts. However, given the decision that prevails in the continuation, the proposer needs only to satisfy the pivotal responder; leaving her indifferent assures that $q-2$ other 
responders will strictly prefer to approve, and all the other players are irrelevant. Our main point is that when decisions are one-dimensional, negotiations are "essentially" twoplayer bargaining problems between the proposer and the pivotal responder. Therefore the condition for uniqueness is "essentially" the Increasing Loss of Delay condition, which assures uniqueness for two-player problems. ${ }^{10}$ We propose it next.

MCD Monotone Compensation for Delay. We say that $u_{i}$ satisfies Monotone Compensation for Delay if the functions $x-\underline{\alpha}_{i}(x)$ and $x-\bar{\alpha}_{i}(x)$ are increasing.

Remark. Single-peakedness assures that $x-\underline{\alpha}_{i}(x)$ is increasing for all $x>p_{i}$, and that $x-\bar{\alpha}_{i}(x)$ is increasing for all $x<p_{i}$. Thus, a single-peaked $u_{i}$ satisfies MCD if (i) the measure of the left acceptance set $\left[\underline{\alpha}_{i}(x), x\right]$ is increasing for $x<p_{i}$; and (ii) the measure of the right acceptance set $\left[x, \bar{\alpha}_{i}(x)\right]$ is decreasing for $x>p_{i}$. In other words, for $x<p_{i}$ (resp. $x>p_{i}$ ) the range of acceptable proposals to the left (right) of $x$ shrinks as $x$ moves away from the peak.

A natural domain of preferences where MCD holds is that of continuous, quasi-concave and concave utilities.

\section{Lemma 4 CONCAVITY IMPLIES MCD.}

(i) A utility function $u_{i}$ that is continuous, strictly quasi-concave and concave satisfies $M C D$.

\footnotetext{
${ }^{10}$ See Osborne and Rubinstein [11]. The main difficulty that remains is that the identity of the relevant "responder", the pivotal voter, depends on the expected continuation outcome.
} 
(ii) A continuous, strictly quasi-concave utility function satisfying MCD may fail to be concave.

See the Appendix for the proof of the first claim. The following example proves the second.

Example 5 Non concave MCD utilities. Consider

$$
\begin{aligned}
u_{i}:[0,1] \rightarrow \Re_{+} \\
u_{i}(x)= \begin{cases}x^{k} & \text { if } x \leq p_{i}, \\
\frac{p_{i}^{k}}{\left(1-p_{i}\right)^{k}}(1-x)^{k} & \text { if } x>p_{i},\end{cases}
\end{aligned}
$$

for $k>1$.

This function is continuous, quasi-concave, satisfies $M C D$, and it is not concave. Consider $x \leq p_{i} \leq y . \quad$ Single-peakedness implies that $x-\bar{\alpha}_{i}(x)$ and $y-\underline{\alpha}_{i}(y)$ are increasing functions. Moreover, it is easy to check that $\underline{\alpha}_{i}(x)=x \delta^{1 / k}$ and $\bar{\alpha}_{i}(y)=1-(1-y) \delta^{1 / k}$. Hence $x-\underline{\alpha}_{i}(x)=\left(1-\delta^{1 / k}\right) x, y-\bar{\alpha}_{i}(y)=(1-y)\left(\delta^{1 / k}-1\right)$ and both are strictly increasing for all $\delta \in(0,1)$.

Our next example shows that without MCD uniqueness fails even for two players.

Example 6 Multiple SSPE When MCD FAils. Let $I=\{1,2\}$ with utilities

$$
\begin{aligned}
& u_{1}(x)=e^{1-x}, \\
& u_{2}(x)=e^{x} .
\end{aligned}
$$


It is immediate to check that MCD fails, and that all pairs $(x, x-\ln \delta), x \in[0,1+\ln \delta]$ are a profile of SSPE proposals.

\subsection{Symmetric utilities}

A straight-forward condition that (with MCD) is sufficient for the uniqueness of the SSPE is symmetry. We will argue that when the agents utilities are symmetric around the peak and satisfy MCD the best response correspondences of all players are contractions. As an SSPE outcome is a fix point of the contraction obtained by a composition of contractions, it must be unique. This holds for every quota $q$.

Proposition 7 Symmetry And MCD ImPly uniqueness For ANY QUOTA. Assume that $\forall i \in I$ utilities are symmetric, $u_{i}(x)=f_{i}\left(\left|p_{i}-x\right|\right)$, and satisfy MCD. Then the SSPE is unique for all $q \geq 2$.

Proof. The proof builds in Lemmata 14, 15 and 16, which are stated and proved in the Appendix. First, Lemma 14 establishes that MCD and symmetry imply that $\underline{\alpha}_{i}$ and $\bar{\alpha}_{i}$ are contractions. Second, Lemma 15 establishes that, for every $q$, when all $\underline{\alpha}_{i}$ and all $\bar{\alpha}_{i}$ are contractions for all $i \in I$ then $\underline{\alpha}$ and $\bar{\alpha}$ are contractions. Third, Lemma 16 establishes that if $\underline{\alpha}$ and $\bar{\alpha}$ are contractions then the best response, $\chi_{i}($.$) , is a contraction$ for every $i \in I$. By Lemmata 14, 15 and 16, and Proposition 3 the equilibrium function $w(x)=\chi_{1}\left(\chi_{2}\left(\ldots \chi_{n-1}\left(\chi_{n}(x)\right)\right)\right)$, being the composition of contractions, is a contraction as well. Consequently, $w(x)=x$ has a unique solution and the result follows. 


\subsection{Qualified majorities and patient players}

Although it is far from being a necessary condition for uniqueness of the SSPE, symmetry cannot be dispensed with in our proof of Proposition 7. Nonetheless, replacing symmetry by a regularity condition on the utilities, we can establish that MCD assures uniqueness when the consensus required is a qualified majority and the agents are sufficiently patient. Our argument crucially relies on the implications of the following observations.

Lemma 8 For $q>n / 2$ and $M C D$ utilities, if $0 \leq x<y \leq 1$, then $x-\chi_{i}(x)<y-\chi_{i}(y)$ for all $i \in I$.

Lemma 9 It is impossible to sustain two different SSPE where the proposals are $\left(x_{1}, \ldots, x_{n}\right)$ and $\left(y_{1}, \ldots, y_{n}\right)$ such that $x_{i+1}-x_{i}<y_{i+1}-y_{i}$ for all $i \in I$.

Lemma 8 is proved in the Appendix. To see that Lemma 9 holds it suffices to observe that if $x_{i+1}-x_{i}<y_{i+1}-y_{i}$ for all $i \in I$, then

$$
\sum_{i=1}^{n-1}\left(x_{i+1}-x_{i}\right)+\left(x_{1}-x_{n}\right)<\sum_{i=1}^{n-1}\left(y_{i+1}-y_{i}\right)+\left(y_{1}-y_{n}\right)
$$

which yields a contradiction.

Consider now two potential SSPE outcomes $x<y$. By Lemma $8, x-\chi_{i}(x)<y-\chi_{i}(y)$ for all $i \in I$. Hence, by Lemma 9, sustaining $x$ and $y$ as SSPE outcomes, requires that the inequality $x=x_{1}<y_{1}=y$ is reversed at the proposals of some player $j>1$, so that $y_{j} \geq x_{j}$. Then, to attain (two) fixed points, another reversal, $y_{j^{\prime}}<x_{j^{\prime}}$, is needed for some $j^{\prime}>j$ (in fact an even number of reversals must occur). To establish the 
uniqueness of SSPE for qualified majorities we will argue that, under MCD and the appropriate regularity condition, either reversals are impossible or they have measures that are incompatible with the existence of more than one SSPE.

When players are sufficiently patient, reversals may occur only when the continuation outcome lies within a small interval of their peak. Thus, the regularity condition that we impose applies to players whose peaks are interior and relevant given $q$. For these players, we will require that, under sufficient patience, when a player expects that an alternative close to her peak prevails in the continuation, unless her best response is precisely her peak, she is not the sole determinant of the acceptance set. To give a formal statement of the requirement, the following notation is used for the relevant subsets of $I$ : $I^{q}=$ $\left\{i \in I \mid 0<p^{n-q+1} \leq p_{i} \leq p^{q}<1\right\}, L_{i}=\left\{j \in I, \mid p_{j}<p_{i}\right\}$, and $R_{i}=\left\{j \in I, \mid p_{j}>p_{i}\right\}$.

REG $\varepsilon$-REGULARIty. Fix $q>n / 2$ and $\varepsilon>0$. We say that the utility profile $\left(u_{1}, . ., u_{n}\right)$ is $\varepsilon$ - regular at $q$ if there exists $\delta_{\varepsilon}$ such that, for any $\delta>\delta_{\varepsilon}$ and all $i \in I^{q}$, either a) $\left(p_{i}-\varepsilon\right)-\underline{\alpha}_{i}\left(p_{i}-\varepsilon\right)>\left(p_{i}+\varepsilon\right)-\underline{\alpha}_{h}\left(p_{i}+\varepsilon\right)$ for all $h \in R_{i}$, and $\bar{\alpha}_{i}\left(p_{i}+\varepsilon\right)-\left(p_{i}+\varepsilon\right)>$
$\bar{\alpha}_{j}\left(p_{i}-\varepsilon\right)-\left(p_{i}-\varepsilon\right)$ for all $j \in L_{i} ;$ or $\left.\mathbf{b}\right) \underline{\alpha}\left(p_{i}+\varepsilon\right) \leq p_{i} \leq \bar{\alpha}\left(p_{i}-\varepsilon\right)$.

The set of players $i \in I^{q}$, for whom condition a) holds is specially relevant in the sequel; we denote it as $\overline{I^{q}}$. Under MCD, a) implies that, for all $z \in\left[p_{i}-\varepsilon, p_{i}+\varepsilon\right]$, $z-\underline{\alpha}_{h}(z)<\left(p_{i}+\varepsilon\right)-\underline{\alpha}_{h}\left(p_{i}+\varepsilon\right)<\left(p_{i}-\varepsilon\right)-\underline{\alpha}_{i}\left(p_{i}-\varepsilon\right) \leq z-\underline{\alpha}_{i}(z)$ for all $h \in R_{i}$, and $\bar{\alpha}_{j}(z)-z>\bar{\alpha}_{i}(z)-z$, for all $j \in L_{i}$. Consequently, for all $i \in \overline{I^{q}}$, the following observation applies: 
R1 For all $z \in\left[p_{i}-\varepsilon, p_{i}+\varepsilon\right]$ and $\delta \geq \delta_{\varepsilon}$,

1. $\underline{\alpha}_{h}(z)>\underline{\alpha}_{i}(z)$ for all $h \in R_{i}$, and

2. $\bar{\alpha}_{j}(z)<\bar{\alpha}_{i}(z)$ for all $j \in L_{i}$.

In fact, $\varepsilon$-regularity and MCD have much stronger implications. When players are sufficiently patient, for a set of continuations $z$ that remain sufficiently close to $p_{i}$, both acceptance bounds of player $i$ remain below those of player $h \in R_{i}$ and above those of players $j \in L_{i}$. This is our next result, which is proved in the Appendix:

Lemma 10 Assume a profile of $M C D$ and $\varepsilon$-regular utilities. If $i \in \overline{I^{q}}$ then there exist $\bar{\varepsilon}, \bar{\delta} \in(0,1)$ such that the following property holds:

$\mathbf{R 2}$ For all $z \in\left[p_{i}-\bar{\varepsilon}, p_{i}+\bar{\varepsilon}\right]$ and $\delta \geq \bar{\delta}$,

1. $\bar{\alpha}_{j}(z)<\bar{\alpha}_{i}(z)<\bar{\alpha}_{h}(z)$ for all $h \in R_{i}, j \in L_{i}$, and

2. $\underline{\alpha}_{h}(z)>\underline{\alpha}_{i}(z)>\underline{\alpha}_{j}(z)$ for all $j \in L_{i}, h \in R_{i}$.

$\mathrm{R} 2$ is very useful to determine what players are relevant for the acceptance set $[\underline{\alpha}(z), \bar{\alpha}(z)]$ when $z$ lies in the neighborhood of a given interior peak $p_{i}, 0<p_{i}<1$. For $p_{i} \in$ $\left(p^{n-q+1}, p^{q}\right)$, it assures that $i$ is irrelevant; for $p_{i}=p^{q}$ or $p_{i}=p^{n-q+1}$, it implies that $\underline{\alpha}=\underline{\alpha}_{i}$ or $\bar{\alpha}=\bar{\alpha}_{i}$, respectively.

We are now ready to establish that sufficient patience assures uniqueness for all $q>$ $n / 2$. 
Proposition 11 Uniqueness FOR QUALIFIEd MAJORITIES AND PATIENT Players. Fix $q>n / 2$ and a profile of $M C D$ utilities where $p_{i} \neq p_{j} \forall i, j \in I$. If $\left(u_{1}, \ldots, u_{n}\right)$ is $\widehat{\varepsilon}$ regular for some $\widehat{\varepsilon}>0$, then there exists $\widehat{\delta} \in(0,1)$ such that, for $\delta \geq \widehat{\delta}$, the SSPE is unique.

The detailed proof is in the Appendix. The outline of the argument is as follows. First, we observe that when agents are sufficiently patient, if two different alternatives are SSPE outcomes, then they must be close to the peak of some player $i$ such that $p^{n-q+1} \leq p_{i} \leq p^{q}$. In the second and third step we assume that $0<p_{i}<1$, and using the implications of $\widehat{\varepsilon}$ - regularity and MCD we derive a contradiction: the second step establishes that $p_{i} \notin\left(p^{n-q+1}, p^{q}\right)$; and the third step rules out that $p_{i}=p^{n-q+1}$ or $p_{i}=p^{q}$. The fourth step takes care of the case $p_{i}=0$ or 1 , (which is relevant only when $q=n$ ) and completes the proof. The assumption that all peaks are different is for convenience and can be relaxed.

The following are examples of environments where the conditions of Proposition 11 are met.

1. MCD utilities, and unanimity.

Under unanimity $I^{q}=\emptyset$, so $\varepsilon$ - regularity is trivially satisfied for all $\delta$ and all $\varepsilon$.

2. MCD utilities, $n$ odd, and simple majority.

The simple majority requirement trivially assures that the regularity condition is met: $q=\frac{n+1}{2}$ implies that $I^{q}=\left\{i \mid p_{i}=p^{n-q+1}=p^{q}\right\}$, and for all $z \in[0,1], \underline{\alpha}(z) \leq$ $p_{i} \leq \bar{\alpha}(z)$ for all $\delta$. 


\section{Differentiable MCD utilities and any qualified majority.}

The differentiability of utilities implies the regularity condition: By differentiability and single peakedness $u_{i}^{\prime}\left(p_{i}\right)=0, u_{h}^{\prime}\left(p_{i}\right)>0$ for $h \in R_{i}$, and $u_{j}^{\prime}\left(p_{i}\right)<0$ for $j \in L_{i}$. It is easy to check that for $p_{i} \in(0,1)$ and $\delta$ sufficiently close to 1 , if $h \in R_{i}$, then $p_{i}-\underline{\alpha}_{i}\left(p_{i}\right)>p_{i}-\underline{\alpha}_{h}\left(p_{i}\right) .{ }^{11}$ By continuity, for $\varepsilon$ sufficiently small $\left(p_{i}-\varepsilon\right)-$ $\underline{\alpha}_{i}\left(p_{i}-\varepsilon\right)>\left(p_{i}+\varepsilon\right)-\underline{\alpha}_{h}\left(p_{i}+\varepsilon\right)$. Similarly, for $j \in L_{i}$, there are $\varepsilon>0$ and $\delta<1$ such that $\bar{\alpha}_{i}\left(p_{i}+\varepsilon\right)-\left(p_{i}+\varepsilon\right)>\bar{\alpha}_{j}\left(p_{i}-\varepsilon\right)-\left(p_{i}-\varepsilon\right)$.

4. MCD utilities that are identical except in the location of the peak, and any qualified majority.

In this case the regularity condition follows by the combination of MCD with identical utilities: Given $q>n / 2$ and an $i \in I^{q}$ there exists $\varepsilon>0$ with $\varepsilon<$ $\varepsilon_{r}=\min \left\{p_{h}-p_{i}: h \in R_{i}\right\}$ such that $u_{i}\left(p_{i}-\varepsilon\right)>u_{h}\left(p_{i}+\varepsilon\right)$ for all $h \in R_{i}$, since $p_{i}+\varepsilon<p_{h}-\varepsilon . \mathrm{MCD}$ implies that $\left(p_{h}-\varepsilon\right)-\underline{\alpha}_{h}\left(p_{h}-\varepsilon\right)>\left(p_{i}+\varepsilon\right)-\underline{\alpha}_{h}\left(p_{i}+\varepsilon\right)$ for all $\delta$. Since utilities are identical $\left(p_{h}-\varepsilon\right)-\underline{\alpha}_{h}\left(p_{h}-\varepsilon\right)=\left(p_{i}-\varepsilon\right)-\underline{\alpha}_{i}\left(p_{i}-\varepsilon\right)$ and therefore $\left(p_{i}+\varepsilon\right)-\underline{\alpha}_{h}\left(p_{i}+\varepsilon\right)<\left(p_{i}-\varepsilon\right)-\underline{\alpha}_{i}\left(p_{i}-\varepsilon\right)$ for all $h \in R_{i}$. Similarly, there exists $\varepsilon<\varepsilon_{l}=\min \left\{p_{i}-p_{j}: j \in L_{i}\right\}$ such that $\bar{\alpha}_{j}\left(p_{i}-\varepsilon\right)-\left(p_{i}-\varepsilon\right)<\bar{\alpha}_{i}\left(p_{i}+\varepsilon\right)-\left(p_{i}+\varepsilon\right)$ for all players $j \in L_{i}$. Thus $\varepsilon$-regularity holds provided that, for all $i \in I^{q}, \varepsilon$ is small enough relative to the $\min \left\{\varepsilon_{r}, \varepsilon_{l}\right\}$.

\footnotetext{
${ }^{11}$ Note that $u_{i}^{\prime}\left(p_{i}\right)=0 \Rightarrow \forall \kappa>0, \exists \delta_{\kappa}$ such that $\frac{u_{i}\left(p_{i}\right)-u_{i}\left(\underline{\alpha}_{i}\left(p_{i}\right)\right)}{p_{i}-\underline{\alpha}_{i}\left(p_{i}\right)}=\frac{u_{i}\left(p_{i}\right)(1-\delta)}{p_{i}-\underline{\alpha}_{i}\left(p_{i}\right)}<\kappa, \forall \delta \geq \delta_{\kappa}$. On the other hand $u_{h}^{\prime}(z)>0 \forall z \leq p_{i} \Rightarrow \exists k>0$ such that $\frac{u_{h}\left(p_{i}\right)-u_{h}\left(\underline{\alpha}_{h}\left(p_{i}\right)\right)}{p_{i}-\underline{\alpha}_{h}\left(p_{i}\right)}=\frac{u_{h}\left(p_{i}\right)(1-\delta)}{p_{i}-\underline{\alpha}_{h}\left(p_{i}\right)}>k, \forall \delta$. Hence, for $\kappa<\frac{u_{i}\left(p_{i}\right)}{u_{h}\left(p_{i}\right)} k$, we have $p_{i}-\underline{\alpha}_{i}\left(p_{i}\right)>p_{i}-\underline{\alpha}_{h}\left(p_{i}\right), \forall \delta \geq \delta_{\kappa}$,
} 
To complete our analysis we now turn attention to the asymptotic uniqueness of SSPE outcomes as the impatience of players vanishes, i.e. $\delta \rightarrow 1$.

\subsection{Asymptotic uniqueness}

Next we establish that for all qualified majorities, $q>n / 2$, as players with MCD preferences become arbitrarily patient, all SSPE outcomes approach to a unique alternative. Furthermore, this convergence holds irrespective of the order by which players make proposals. Thus, for environments with very patient players we can give a unique (limit) prediction that depends only on the configuration of individual preferences and the qualified majority required. The intuition behind this result is simple: given $q$ and for $\delta$ sufficiently high, only equilibrium proposals that are very close to $p^{n-q+1}$ or $p^{q}$, say in $\left(p^{n-q+1}-\varepsilon, p^{n+q-1}+\varepsilon\right) \cap[0,1]$ or $\left(p^{q}-\varepsilon, p^{q}+\varepsilon\right) \cap[0,1]$, might induce reversals in the best responses (which, by Lemmata 8 and 9, are necessary to sustain multiple SSPE). However, as patience increases these (disjoint) intervals shrink and $\left|\chi_{i}(x)-x\right|$ becomes arbitrarily small for all $i \in I$; thus, even if reversals in the best responses cannot be ruled out, their impact is negligible.

Proposition 12 Asymptotic Uniqueness for Qualified majorities. Let $q>n / 2$ and assume that preferences satisfy $M C D$. For all $\varepsilon>0$ there is $\delta_{\varepsilon} \in(0,1)$ such that if $x_{1}$ and $y_{1}$ are SSPE outcomes for $\delta \geq \delta_{\varepsilon}$, then $\left|x_{1}-y_{1}\right|<\varepsilon$.

Proof. Lemma 18, establishes that when players are sufficiently patient, all the proposals $x_{i}$ in a given SSPE must be nearby the initial proposal (and equilibrium outcome) $x_{1}$. 
We now combine Lemma 18 with MCD to establish that as $\delta$ approaches 1 all SSPE outcomes converge. Assume there is some $\varepsilon>0$ such that for all $\delta \in(0,1)$ there are two SSPE outcomes with proposal profiles $x=\left(x_{1}, \ldots, x_{n}\right)$ and $y=\left(y_{1}, \ldots, y_{n}\right)$ such that $x_{1}+$ $\varepsilon / 2<y_{1}-\varepsilon / 2$. (Note that the protocol at which $x$ and $y$ are SSPE need not be the same, identifying the first proposer as player 1 is just for convenience).

Given $\varepsilon>0$, by Lemma 18 there is a $\delta_{\varepsilon}$, such that, for $\delta \geq \delta_{\varepsilon}$, all $x_{i} \in\left(x_{1}-\varepsilon / 2, x_{1}+\varepsilon / 2\right) \cap$ $[0,1]$ and all $y_{i} \in\left(y_{1}-\varepsilon / 2, y_{1}+\varepsilon / 2\right) \cap[0,1]$. Since $x_{k}<y_{j}$ for all $x_{k}$ and $y_{j}$, by Lemma 8, $x_{k}-\chi_{i}\left(x_{k}\right)<y_{j}-\chi_{i}\left(y_{j}\right)$ for all $i, j, k \in I$. Therefore, using Lemma 9 we get a contradiction.

Since the argument carries if the (deterministic) protocols by which $x$ and $y$ are the SSPE outcomes are different, the following corollary is immediate.

Corollary 13 Asymptotic Protocol Independence for Qualified Majorities. Given a profile of $M C D$ utilities and a qualified majority $q>n / 2$, all SSPE converge to the same outcome independently of the order of play.

Remark. The asymptotic independence of the protocol clearly fails in games where decisions need only the approval of a minority. The intuition is simple: proposers always seek the support of their neighbors. When only a small number of votes is necessary $(q \leq n / 2)$, there is no subset of players whose vote is needed by every proposer; therefore equilibrium outcomes may change drastically depending on the order by which players propose. 


\section{Conclusions}

We have explored multilateral bargaining on a one-dimensional characteristic of a public good when decisions require the favorable vote of at least $q$ participants under a deterministic protocol of alternating proposals. For the full range of quota requirements (ranging from minority consensus to unanimity) we provided a characterization of SSPE, and we establish sufficient conditions assuring uniqueness.

When decisions require a consensus weaker than unanimity, assuming that everyone gains in every agreement is an important limitation of our analysis, since it excludes environments where decisive coalitions may impose alternatives at which some players are worse off than in perpetual disagreement. Unfortunately, addressing these scenarios poses difficulties that we could not resolve, because neither existence nor uniqueness can be assured.

Our results provide a useful tool to address issues of coalition formation. Since the provision of public goods is a main motivation fueling group formation and segregation, precise predictions on bargaining within groups are fundamental to discuss the formation and stability of coalitions. We plan to explore this in future research.

\section{References}

[1] J.S. Banks, J. Duggan, A Bargaining Model of Collective Choice, Amer. Polit. Sci. Rev. 94 (2000), 73-88. 
[2] K. Chatterjee, B. Dutta, K. Sengupta, A Noncooperative Theory of Coalitional Bargaining, Rev. Econ. Stud. 60 (1993), 463-477.

[3] S. Cho, J. Duggan, Uniqueness of stationary equilibria in a one-dimensional model of bargaining, J. Econ. Theory 113 (2003),118-130.

[4] H. Eraslan, Uniqueness of stationary equilibrium payoffs in the Baron-Ferejohn model, J. Econ. Theory 103 (2002),11-30.

[5] R. Evans, Coalitional Bargaining with Competition to Make Offers, Games Econ. Behav. 19 (1997), 211-220.

[6] M. O. Jackson, B. Moselle, Coalition and Party Formation in a legislative Voting Game, J. Econ. Theory 103 (2002), 49-87.

[7] S. Hart, A. Mas-Colell, Bargaining and Value, Econometrica 64 (1996), 357-380.

[8] B. Moldovanu, E. Winter, Order Independent Equilibria, Games Econ. Behav. 9 (1995), 21-34.

[9] H. Moulin, On Strategy-Prooffness and Single Peakedness, Public Choice 35 (1980), $437-455$

[10] H. Moulin, Axioms of Cooperative Decision Making, Cambridge Univ. Press, Cambridge, 1988.

[11] M. Osborne, A. Rubinstein, Bargaining and Markets, Academic Press, San Diego, 1990. 
[12] M. Perry, P. Reny, A Noncooperative View of Coalition Formation and the Core, Econometrica 62 (1994), 795-817.

[13] A. Rubinstein, Perfect Equilibrium in a Bargaining Model, Econometrica 50 (1982), 97-109.

[14] R. Selten, A Noncooperative Model of Characteristic Function Bargaining, in: V. Bohm and H. Nachtkamp (Eds.), Essays in Game Theory and Mathematical Economics in Honour of Oskar Morgenstern, Bibliographisches Institut, Mannheim, 1981.

\section{Appendix}

Notation. The following notational conventions are used.

1. $x, y, z$ denote generic alternatives in $[0,1] . \mathbf{x}, \mathbf{y}, \mathbf{z}$, denote generic profiles of alternatives; whenever we refer to $\mathbf{x}=\left(x_{1}, \ldots, x_{n}\right)$ as an SSPE profile of proposals we write $x$ to refer to the corresponding equilibrium outcome.

2. Given utility function $u$ with peak at $p$ and alternative $z \in[0,1]$, and its associated left and right functions $r$ and $l$ (defined in Section 3), we denote by $\lambda(z)$ and $\rho(z)$ the following two alternatives:

$$
\lambda(z)=\left\{\begin{array}{cc}
l^{-1}(u(z)) & \text { if } u(z) \in[u(0), u(p)] \\
0 & \text { otherwise }
\end{array}\right.
$$


and

$$
\rho(z)=\left\{\begin{array}{cc}
r^{-1}(u(z)) & \text { if } u(z) \in[u(p), u(1)] \\
1 & \text { otherwise. }
\end{array}\right.
$$

That is, $\lambda(z)$ (resp $\rho(z))$ is the alternative to the left (right) of $p$ that gives the feasible utility closest to $u(z)$; thus, for $z \leq p, \lambda(z)=z$, and $\rho(z)=z$ for $z \geq p$. Subindexed $\lambda_{i}$ and $\rho_{i}$ will be used if necessary.

\section{Proof of Proposition 3.}

Claim: There is no SSPE where the players use strictly mixed strategies.

Consider a profile of stationary strategies. Player $i$ 's strategy specifies her proposal and her acceptance/rejection rule to the opponent's proposals. Strategies are history independent; thus proposals are independent of past play and acceptance/rejection decisions depend only on the standing proposal and, possibly, on who the proposer is.

By stationarity, the continuation expected outcome $x$ is independent on the actions of the players in the current period. Given this expected outcome, the proposer can choose among a set of outcomes which is a closed interval $[\underline{\alpha}(x), \bar{\alpha}(x)] \subseteq[0,1]$. Moreover, the utilities of the players are continuous an strictly quasi-concave so that there is a unique optimal proposal for any interval $[\underline{\alpha}(x), \bar{\alpha}(x)]$.

Let us now check that the strategy cannot be mixed in the stage of acceptance. This could be only when some player is indifferent between continuation and the proposal. If some player rejects the proposal with positive probability, then it will be in the interest of the proposer to increase the utility of this player by changing marginally her proposal 
so that she accepts it. This is possible if no other player gets a payoff equal or smaller than her continuation utility. However, this proposal exists for any continuation payoff (since $\delta \in(0,1))$.

\section{Proof of Lemma 4(i).}

We prove that if $u_{i}=u$ is continuous, strictly quasi-concave and concave then $x-\underline{\alpha}_{i}(x)$ is increasing for all $x \in[0,1]$. A symmetric argument proves that $x-\bar{\alpha}_{i}(x)$ is increasing. (In what follows we drop subindex $i$ except in $\underline{\alpha}_{i}$ and $\bar{\alpha}_{i}$.)

Since $u$ is continuous, strictly quasi-concave and concave, it has a unique maximum. Given, $x, x^{\prime} \in[0,1], x<x^{\prime}$, we distinguish three cases depending on the position of $x, x^{\prime}$ relative to the peak $p$ :

1. Let $p \leq x<x^{\prime}$. In this case the desired inequality holds without appealing to concavity: Since $u(x)>u\left(x^{\prime}\right)$ then $\underline{\alpha}_{i}(x) \geq \underline{\alpha}_{i}\left(x^{\prime}\right)$, and it is immediate that $x-\underline{\alpha}_{i}(x)<x^{\prime}-\underline{\alpha}_{i}\left(x^{\prime}\right)$.

2. Let $x<x^{\prime} \leq p$. In this case, $(1-\delta) u(x)=u(x)-\delta u(x)<u\left(x^{\prime}\right)-\delta u\left(x^{\prime}\right)=$ $(1-\delta) u\left(x^{\prime}\right)$

(a) Assume that $\delta u(x) \in[u(0), u(p)]$, which implies $\delta u\left(x^{\prime}\right) \in[u(0), u(p)]$. Observe that $\delta u(z)=u\left(l^{-1}(\delta u(z))\right)$ since, for any $z \in[0, p], l(z)=u(z)$. Hence,

$$
u(x)-u\left(l^{-1}(\delta u(x))\right)<u\left(x^{\prime}\right)-u\left(l^{-1}\left(\delta u\left(x^{\prime}\right)\right)\right)
$$

The concavity of $u$, implies that

$$
u(x)-u(x-a) \geq u\left(x^{\prime}\right)-u\left(x^{\prime}-b\right) \text { for any } a \geq b .
$$


Rewrite $l^{-1}\left(\delta u\left(x^{\prime}\right)\right)=x^{\prime}-b$ and $l^{-1}(\delta u(x))=x-a$, and use the previous inequalities to conclude that $a<b$. Hence, $x-\underline{\alpha}_{i}(x)=x-l^{-1}(\delta u(x))<$ $x^{\prime}-l^{-1}\left(\delta u\left(x^{\prime}\right)\right)=x^{\prime}-\underline{\alpha}_{i}\left(x^{\prime}\right)$.

(b) Assume $\delta u(x) \notin[u(0), u(p)]$, so that $\underline{\alpha}_{i}(x)=0$, and $\delta u\left(x^{\prime}\right) \in\left[u(0), u\left(p_{i}\right)\right]$. Consider $y \in\left[x, x^{\prime}\right]$ such that $\delta u(y)=u(0)$ and use (a) to conclude that $x-\underline{\alpha}_{i}(x)=x<y=y-l^{-1}(\delta u(y))<x^{\prime}-l^{-1}\left(\delta u\left(x^{\prime}\right)\right)=x^{\prime}-\underline{\alpha}\left(x^{\prime}\right)$.

(c) If $\delta u(x) \notin[u(0), u(p)]$ and $\delta u\left(x^{\prime}\right) \notin[u(0), u(p)]$ then $\underline{\alpha}_{i}(x)=0$ and $\underline{\alpha}_{i}\left(x^{\prime}\right)=$ 0 and obviously $x-\underline{\alpha}_{i}(x)=x<x^{\prime}=x^{\prime}-\underline{\alpha}_{i}\left(x^{\prime}\right)$.

3. Let $x<p<x^{\prime}$. Now consider $\lambda\left(x^{\prime}\right)$ (recall that $\lambda\left(x^{\prime}\right)=l^{-1}\left(u\left(x^{\prime}\right)\right)$ if $u\left(x^{\prime}\right) \in$ $[u(0), u(p)]$, and $\lambda\left(x^{\prime}\right)=0$ otherwise) and observe that $\underline{\alpha}_{i}\left(\lambda\left(x^{\prime}\right)\right)=\underline{\alpha}_{i}\left(x^{\prime}\right)$. If $x<$ $\lambda\left(x^{\prime}\right) \leq p$ then the desired inequality follows from Case 2:

$$
x-\underline{\alpha}_{i}(x)<\lambda\left(x^{\prime}\right)-\underline{\alpha}_{i}\left(\lambda\left(x^{\prime}\right)\right)<x^{\prime}-\underline{\alpha}_{i}\left(x^{\prime}\right) .
$$

If $\lambda\left(x^{\prime}\right) \leq x<p$, then $\underline{\alpha}_{i}(x) \geq \underline{\alpha}_{i}\left(\lambda\left(x^{\prime}\right)\right)=\underline{\alpha}_{i}\left(x^{\prime}\right)$, and therefore

$$
x-\underline{\alpha}_{i}(x)<x^{\prime}-\underline{\alpha}_{i}\left(x^{\prime}\right) .
$$

Hence, concavity implies MCD.

\section{Proof of Lemma 8.}

STEP 1 . We show that $0 \leq x<y \leq 1$ implies that $y-\underline{\alpha}(y)>x-\underline{\alpha}(x)$ and $y-\bar{\alpha}(y)>$ $x-\bar{\alpha}(x)$.

What follows proves the first implication; a similar argument proves the second.

Note that for any $x, y, 0 \leq x<y \leq 1$ the following observations hold: 
(i) There is a subset of the players, $Q \subset I$, \#Q $\geq n-q+1$, such that $\underline{\alpha}_{j}(y) \geq \underline{\alpha}(y)$ for all $j \in Q$. This follows directly from the definition of $\underline{\alpha}(y)$.

(ii) MCD implies that $y-\underline{\alpha}_{i}(y)>x-\underline{\alpha}_{i}(x)$ for all $i \in I$.

To establish the claimed inequality we proceed by contradiction. Assume that $y-$ $\underline{\alpha}(y) \leq x-\underline{\alpha}(x)$. Then $\underline{\alpha}(x)<\underline{\alpha}_{j}(x)$ for all $j \in Q$, since otherwise, (i) implies that

$$
y-\underline{\alpha}_{j}(y) \leq y-\underline{\alpha}(y) \leq x-\underline{\alpha}(x) \leq x-\underline{\alpha}_{j}(x) \text { for some } j \in Q,
$$

which contradicts (ii). However, since $\# Q \geq n-q+1, \underline{\alpha}(x)$ must be an acceptable alternative when the continuation outcome is $x$ for least one player in $Q$. Therefore $\underline{\alpha}(x) \geq$ $\min \left\{\underline{\alpha}_{j}(x): j \in Q\right\}$, which is also a contradiction.

Step 2. For any $x, y, 0 \leq x<y \leq 1$, we examine all possible values of the best response to $x, \chi_{i}(x)$, to establish that $y-\chi_{i}(y)>x-\chi_{i}(x)$ :

(i) $\chi_{i}(x)=\bar{\alpha}(x)$. Using Step 1 and the definition of $\chi_{i}(\cdot), y-\chi_{i}(y) \geq y-\bar{\alpha}(y)>$ $x-\bar{\alpha}(x)=x-\chi_{i}(x)$.

(ii) $\chi_{i}(x)=p_{i}$. If $\chi_{i}(y)=p_{i}$, the claim follows directly. Otherwise, either $\chi_{i}(y)=$ $\bar{\alpha}(y)$ or $\chi_{i}(y)=\underline{\alpha}(y)$. If $\chi_{i}(y)=\bar{\alpha}(y)$, then $\bar{\alpha}(y) \leq p_{i} \leq \bar{\alpha}(x)$ since $p_{i} \in[\underline{\alpha}(x), \bar{\alpha}(x)]$. Hence, $y-\chi_{i}(y)=y-\bar{\alpha}(y) \geq y-p_{i}>x-p_{i}=x-\chi_{i}(x)$. If $\chi_{i}(y)=\underline{\alpha}(y)$, using Step 1 and the fact that $p_{i} \in[\underline{\alpha}(x), \bar{\alpha}(x)]$ we get $y-\chi_{i}(y)=y-\underline{\alpha}(y)>x-\underline{\alpha}(x) \geq x-p_{i}=x-\chi_{i}(x)$.

(iii) $\chi_{i}(x)=\underline{\alpha}(x)$. We distinguish three subcases: if $\chi_{i}(y)=\underline{\alpha}(y)$ the result follows directly from Step 1. The case $\chi_{i}(y)=p_{i}$ can occur only if $\underline{\alpha}(y) \leq p_{i} \leq \underline{\alpha}(x)$ since $p_{i} \in[\underline{\alpha}(y), \bar{\alpha}(y)]$ and $p_{i} \leq \underline{\alpha}(x)$. Therefore, $y-\chi_{i}(y)=y-p_{i}>x-p_{i} \geq x-\underline{\alpha}(x)=$ 
$x-\chi_{i}(x)$. The last possibility is that $\chi_{i}(y)=\bar{\alpha}(y)$, which occurs when $\bar{\alpha}(y) \leq p_{i} \leq \underline{\alpha}(x)$. Therefore, $y-\chi_{i}(y)=y-\bar{\alpha}(y) \geq y-p_{i}>x-p_{i} \geq x-\underline{\alpha}(x)=x-\chi_{i}(x)$.

\section{Proof of Lemma 10.}

Let $D=\min \left\{\left|p_{i}-p_{j}\right|: i, j \in I, p_{i} \neq p_{j}\right\}$. Take an $\bar{\varepsilon}<\min \{\varepsilon, D / 2\}$ such that if $z \in$ $\left[p_{i}-\bar{\varepsilon}, p_{i}+\bar{\varepsilon}\right]$ then both $\lambda_{i}(z), \rho_{i}(z) \in\left[p_{i}-D / 2, p_{i}+D / 2\right]$.

Recall observation R1, i.e. for all $\delta \geq \delta_{\varepsilon}, \bar{\alpha}_{i}(z)>\bar{\alpha}_{j}(z)$ for all $j \in L_{i}$ and $\underline{\alpha}_{i}(z)<$ $\underline{\alpha}_{h}(z)$ for all $h \in R_{i}$. To prove R2-1, we need to show that there exists $\bar{\delta} \in(0,1), \bar{\delta} \geq \delta_{\varepsilon}$, such that for all $\delta>\bar{\delta}$ if $z \in\left[p_{i}-\bar{\varepsilon}, p_{i}+\bar{\varepsilon}\right]$ then $\bar{\alpha}_{h}(z)>\bar{\alpha}_{i}(z)$ for all $h \in R_{i}$. Consider an $h \in R_{i}$, and note that single-peakedness implies $\bar{\alpha}_{h}(z) \geq p_{h}$ for all $\delta \in(0,1)$. There exists $\widetilde{\delta} \in(0,1)$ such that for all $\delta \geq \widetilde{\delta}$, by definition $\bar{\alpha}_{i}(z)=\bar{\alpha}_{i}\left(\rho_{i}(z)\right)=r_{i}^{-1}\left(\delta u_{i}\left(\rho_{i}(z)\right)\right)$, and $r_{i}^{-1}\left(u_{i}\left(\rho_{i}(z)\right)\right)=\rho_{i}(z)$ (note that $\widetilde{\delta}$ can be selected to guarantee that $\delta u_{i}\left(\rho_{i}(z)\right) \in$ $\left[u_{i}(1), u_{i}\left(p_{i}\right)\right]$ for all $\delta \geq \widetilde{\delta}$, and thus $r^{-1}$ is well defined). For $z \in\left[p_{i}-\bar{\varepsilon}, p_{i}+\bar{\varepsilon}\right]$ fixed, consider the difference $r_{i}^{-1}\left(\delta u_{i}\left(\rho_{i}(z)\right)\right)-r_{i}^{-1}\left(u_{i}\left(\left(\rho_{i}(z)\right)\right)\right)$ for $\delta \geq \widetilde{\delta}$. This difference is a continuous function of $\delta$, which has value zero at $\delta=1$. Hence, there exists $\bar{\delta} \geq$ $\max \left\{\delta_{\varepsilon}, \widetilde{\delta}\right\}$ such that

$$
r_{i}^{-1}\left(\delta u_{i}\left(\rho_{i}(z)\right)\right)-r_{i}^{-1}\left(u_{i}\left(\left(\rho_{i}(z)\right)\right)\right)=\bar{\alpha}_{i}\left(\rho_{i}(z)\right)-\rho_{i}(z)<\bar{\varepsilon} \text { for all } \delta>\bar{\delta},
$$

and, since $\rho_{i}(z)<p_{i}+D / 2$,

$$
\bar{\alpha}_{i}(z)=\bar{\alpha}_{i}\left(\rho_{i}(z)\right)<p_{i}+D / 2+\bar{\varepsilon}<p_{i}+D \leq p_{h} \leq \bar{\alpha}_{h}(z) \text { for all } \delta>\bar{\delta} .
$$

$\mathrm{R} 2-2$ is proved analogously. 


\section{Proof of Proposition 11.}

Some preliminary remarks are in order. By Lemma 10 we can select $\bar{\varepsilon}, \bar{\delta} \in(0,1), \bar{\varepsilon}<$ $\min \{\widehat{\varepsilon}, D / 2\}, D=\min _{i, j \in I}\left\{\left|p_{i}-p_{j}\right|: p_{i} \neq p_{j}\right\}$, such R2 holds for all $i \in \overline{I^{q}}$. On the other hand, by Lemma 20, $\bar{\varepsilon}$ and $\bar{\delta}$ can be selected so that, in addition to R2 for all $i \in \overline{I^{q}}$, the following technical property (that follows by continuity and single-peakness) is also assured for all $i \in I$ :

SIGN: Either $\left|\lambda_{i}(x)-\lambda_{i}(y)\right| \leq\left|\rho_{i}(x)-\rho_{i}(y)\right|$ for all $x, y \in[p-\bar{\varepsilon}, p+\bar{\varepsilon}]$, or the reverse weak inequality holds throughout this interval.

Next we prove the proposition in four steps.

STEP 1. Claim: There exist $\delta_{1} \in(0,1)$ such that, $\forall \delta>\delta_{1}$, if $\mathbf{x}$ and $\mathbf{y}$ are SSPE with

outcomes $x<y$ then there is some $p_{i} \in\left[p^{n-q+1}, p^{q}\right]$ such that $x_{j}, y_{j} \in\left[p_{i}-\bar{\varepsilon}, p_{i}+\bar{\varepsilon}\right] \cap[0,1]$ for all $j \in I$.

By Lemma 18, for $\bar{\varepsilon} / 4>0$, there is a $\delta_{1} \geq \delta_{\widehat{\varepsilon}}$ (where $\delta_{\widehat{\varepsilon}}<1$ is the $\delta$ lower bound for $\widehat{\varepsilon}$-regularity), such that if $\delta>\delta_{1}$ and $\mathbf{z}$ is an SSPE, then $z_{i} \in(z-\bar{\varepsilon} / 4, z+\bar{\varepsilon} / 4) \forall i \in I$. Fix $\delta>\delta_{1}$ and assume that $\mathbf{x}$ and $\mathbf{y}$ are SSPE with outcomes $x<y$. By Lemma 19, $|x-y|<\bar{\varepsilon} / 2$ and $(x-\bar{\varepsilon} / 4, y+\bar{\varepsilon} / 4) \cap\left\{p_{1}, \ldots, p_{n}\right\}=p_{i}$. Since $p_{i} \in(x-\bar{\varepsilon} / 4, y+\bar{\varepsilon} / 4)$ and $z_{i} \in(x-\bar{\varepsilon} / 4, y+\bar{\varepsilon} / 4)$ for $z=x, y$, it follows that

$$
z_{i}-p_{i} \leq y+\bar{\varepsilon} / 4-[x-\bar{\varepsilon} / 4]=y-x+\bar{\varepsilon} / 2<\bar{\varepsilon},
$$

and

$$
z_{i}-p_{i} \geq x-\bar{\varepsilon} / 4-[y+\bar{\varepsilon} / 4]=-(y-x)-\bar{\varepsilon} / 2>-\bar{\varepsilon} / 2-\bar{\varepsilon} / 2=-\bar{\varepsilon} ;
$$


and therefore all proposals in $\mathbf{x}$ and $\mathbf{y}$ lie in $\left[p_{i}-\bar{\varepsilon}, p_{i}+\bar{\varepsilon}\right] \cap[0,1]$.

We can rule out that $p_{i} \in\left[0, p^{n-q+1}\right) \cup\left(p^{q}, 1\right]$ : Otherwise, there is a player $j$ with $p_{j} \in\left[p^{n-q+1}, p^{q}\right]$ that proposes $x_{j}=y_{j}=p_{j}$ in both equilibria (since it is acceptable at both alleged equilibrium continuations); this contradicts that $x<y$. Hence, $p_{i} \in\left[p^{n-q+1}, p^{q}\right]$.

Before we proceed to Step 2, note that for $i \in I^{q}$ the hypothesis that $\mathbf{x}$ and $\mathbf{y}$ are SSPE with outcomes $x, y \in\left[p_{i}-\widehat{\varepsilon}, p_{i}+\widehat{\varepsilon}\right], x<y$, rules out that $\underline{\alpha}\left(p_{i}+\widehat{\varepsilon}\right) \leq p_{i} \leq \bar{\alpha}\left(p_{i}-\widehat{\varepsilon}\right)$, i.e. condition $\mathbf{b}$ ) in the definition of $\widehat{\varepsilon}$-regularity. The reason is simple; under $\mathbf{b}$ ) the equilibrium proposal of player $i$ ought to be the same at both SSPE, contradicting that $x<y$. Hence, in what follows, $\widehat{\varepsilon}$-regularity implies condition a). Thus, given the peak identified in Step 1, either $0<p^{n-q+1} \leq p_{i} \leq p^{q}<1$ which implies that $i \in \overline{I^{q}}$ and R2 applies; or else, $q=n$ and $p_{i}=p^{1}=0$ or $p_{i}=p^{n}=1$.

In Steps 2 and 3, we maintain the assumption that $0<p^{n-q+1} \leq p_{i} \leq p^{q}<1$. In Step 4 , we take care of the cases where $p_{i}=p^{q}=0$, or $p_{i}=p^{n}-q+1=1$.

SteP 2. Claim: Let $p_{i} \in(0,1)$. There exist $\delta_{2} \in(0,1)$ such that, $\forall \delta \geq \delta_{2}$, if $\mathbf{x}$ and $\mathbf{y}$ are SSPE with outcomes $x<y$ and $x_{j}, y_{j} \in\left[p_{i}-\bar{\varepsilon}, p_{i}+\bar{\varepsilon}\right]$ for all $j \in I$, then $p_{i} \notin\left(p^{n-q+1}, p^{q}\right)$.

We will establish that the claim holds for $\delta_{2}=\max \left\{\delta_{1}, \bar{\delta}\right\}$. Assume $\delta \geq \delta_{2}$, which implies R2, and let $p_{i} \in\left(p^{n-q+1}, p^{q}\right)$. By R2, $\underline{\alpha}_{h}(z)>\underline{\alpha}_{i}(z)$ and $\underline{\alpha}_{h}(z)>\underline{\alpha}_{j}(z)$ for all $z \in\left[p_{i}-\bar{\varepsilon}, p_{i}+\bar{\varepsilon}\right]$ for all $j \in L_{i}$ and all $h \in R_{i}$. Moreover, $p_{i}<p^{q}$ implies that $\# R_{i} \geq n-q+1$, so that, by Lemma $21, \underline{\alpha}$ is a contraction in $\left[p_{i}-\bar{\varepsilon}, p_{i}+\bar{\varepsilon}\right]$. Similarly, 
we can argue that $\bar{\alpha}$ is a contraction in $\left[p_{i}-\bar{\varepsilon}, p_{i}+\bar{\varepsilon}\right]$. Applying Lemma 16 we conclude that the best responses of all players are contractions in $\left[p_{i}-\bar{\varepsilon}, p_{i}+\bar{\varepsilon}\right]$, implying that at most one equilibrium can be attained in this interval, which is a contradiction.

SteP 3. Claim: Let $p_{i} \in(0,1)$ and $\delta \geq \delta_{2}$. If $\mathbf{x}$ and $\mathbf{y}$ are SSPE with outcomes $x<y$ and $x_{j}, y_{j} \in\left[p_{i}-\bar{\varepsilon}, p_{i}+\bar{\varepsilon}\right]$ for all $j \in I$, then neither $p_{i}=p^{n-q+1}$ nor $p_{i}=p^{q}$. We prove that $p_{i} \neq p^{n-q+1}$; the proof that $p_{i} \neq p^{q}$ is analogous.

Since $\delta_{2} \geq \bar{\delta}$, we establish the claim for each of the two possible cases allowed by SIGN.

Case 1: $\left|\lambda_{i}(x)-\lambda_{i}(y)\right| \geq\left|\rho_{i}(x)-\rho_{i}(y)\right|$ for all $x, y \in\left[p_{i}-\bar{\varepsilon}, p_{i}+\bar{\varepsilon}\right]$.

For this case, we can establish that $\underline{\alpha}$ and $\bar{\alpha}$ are contractions in $\left[p^{n-q+1}-\bar{\varepsilon}, p^{n-q+1}+\bar{\varepsilon}\right]$ and apply Lemma 16 to obtain a contradiction. Since $p_{i}=p^{n-q+1}<p^{q}$, the same argument of Step 2 applies to see that $\underline{\alpha}$ is a contraction. To complete the proof of the claim for this case, we show that $\bar{\alpha}$ is a contraction. Note that \# $\left(L_{i} \cup\{i\}\right)=n-q+1$, so that $\mathrm{R} 2$ implies that $\bar{\alpha}_{i}=\bar{\alpha}$. Hence it will suffice to prove that $\bar{\alpha}_{i}$ is a contraction in this interval. Assume w.l.o.g. $\rho_{i}(x)<\rho_{i}(y)$. Then, by $\operatorname{MCD} \bar{\alpha}_{i}\left(\rho_{i}(x)\right)-\rho_{i}(x)>$ $\bar{\alpha}_{i}\left(\rho_{i}(y)\right)-\rho_{i}(y)$ and, by single-peakedness, $\bar{\alpha}_{i}\left(\rho_{i}(x)\right) \leq \bar{\alpha}_{i}\left(\rho_{i}(y)\right)$. Thus,

$$
\left|\bar{\alpha}_{i}\left(\rho_{i}(y)\right)-\bar{\alpha}_{i}\left(\rho_{i}(x)\right)\right|<\left|\rho_{i}(y)-\rho_{i}(x)\right| .
$$


Note as well that that, in the present case, $\left|\rho_{i}(y)-\rho_{i}(x)\right| \leq|y-x| .{ }^{12}$ Now, using $\left|\bar{\alpha}_{i}\left(\rho_{i}(y)\right)-\bar{\alpha}_{i}\left(\rho_{i}(x)\right)\right|=\left|\bar{\alpha}_{i}(y)-\bar{\alpha}_{i}(x)\right|$ we conclude that $\left|\bar{\alpha}_{i}(y)-\bar{\alpha}_{i}(x)\right|<|y-x|$.

Case $2\left|\lambda_{i}(x)-\lambda_{i}(y)\right| \leq\left|\rho_{i}(x)-\rho_{i}(y)\right| \forall x, y \in\left[p_{i}-\bar{\varepsilon}, p_{i}+\bar{\varepsilon}\right]$.

Presently $\bar{\alpha}$ may not be a contraction; so we need a direct contradiction to the hypothesis that two different SSPE exist. Departing from this hypothesis, note that, at least in one of the two alleged SSPE, say in $\mathbf{x}$, the proposal of agent $i$ must be different from her peak, $x_{i} \neq p_{i}=p^{n-q+1}$. Thus, $\mathrm{x}$ is such that $x_{h}=\bar{\alpha}\left(x_{h+1}\right)$ for $h \in R_{i}, x_{j}=\underline{\alpha}\left(x_{j+1}\right)$ for $j \in L_{i}$, and either $x_{i}=\underline{\alpha}\left(x_{i+1}\right)$ or $x_{i}=\bar{\alpha}\left(x_{i+1}\right)$. Assume, w.l.o.g., the first possibility, $x_{i}=\underline{\alpha}\left(x_{i+1}\right)$. There exist $a_{j} \geq 0$ and $b_{h} \geq 0$ such that

$$
x_{j}=\underline{\alpha}\left(x_{j+1}\right)=x_{j+1}-a_{j} \text { for } j \in L_{i} \cup\{i\} \text { and } x_{h}=\bar{\alpha}\left(x_{h+1}\right)=x_{h+1}+b_{h} \text { for } h \in R_{i} .
$$

Also, note that for any $z, w \in\left[p_{i}-\bar{\varepsilon}, p_{i}+\bar{\varepsilon}\right]$ there is an $h \in R_{i}$ such that $\underline{\alpha}(z)=\underline{\alpha}_{h}(z)$ and by $\widehat{\varepsilon}$-regularity and MCD

$$
\begin{aligned}
z-\underline{\alpha}_{h}(z) & \leq\left(p_{i}+\bar{\varepsilon}\right)-\underline{\alpha}_{h}\left(p_{i}+\bar{\varepsilon}\right)<\left(p_{i}+\widehat{\varepsilon}\right)-\underline{\alpha}_{h}\left(p_{i}+\widehat{\varepsilon}\right) \\
& <\left(p_{i}-\widehat{\varepsilon}\right)-\underline{\alpha}_{i}\left(p_{i}-\widehat{\varepsilon}\right)<\left(p_{i}-\bar{\varepsilon}\right)-\underline{\alpha}_{i}\left(p_{i}-\bar{\varepsilon}\right) \leq w-\underline{\alpha}_{i}(w),
\end{aligned}
$$

so that

$$
z-\underline{\alpha}(z)<w-\underline{\alpha}_{i}(w) \text { for all } z, w \in\left[p_{i}-\bar{\varepsilon}, p_{i}+\bar{\varepsilon}\right]
$$

Next, observe that if $z, \bar{\alpha}(z) \in\left[p_{i}-\bar{\varepsilon}, p_{i}+\bar{\varepsilon}\right]$ then $\underline{\alpha}(z), \lambda_{i}(z) \in\left[p_{i}-\bar{\varepsilon}, p_{i}+\bar{\varepsilon}\right]$. Recall that since $\#\left(L_{i} \cup\{i\}\right)=n-q+1$, R2 assures that $\bar{\alpha}(z)=\bar{\alpha}_{i}(z)$ and by definition

\footnotetext{
${ }^{12}$ To check this, consider all possible cases: (i) $x=\rho_{i}(x), y=\rho_{i}(y)$, (ii) $x=\rho_{i}(x), y=\lambda_{i}(y)$, (iii) $x=\lambda_{i}(x), y=\rho_{i}(y)$ and (iv) $x=\lambda_{i}(x), y=\lambda_{i}(y)$, and use $\left|\lambda_{i}(x)-\lambda_{i}(y)\right| \geq\left|\rho_{i}(x)-\rho_{i}(y)\right|$, $\rho_{i}(x)<\rho_{i}(y)$ and (by single-peakedeness) $\lambda_{i}(x) \geq \lambda_{i}(y)$.
} 
$\lambda_{i}\left(p_{i}\right)=\rho_{i}\left(p_{i}\right)=p_{i}$. To check that $\underline{\alpha}(z) \in\left[p_{i}-\bar{\varepsilon}, p_{i}+\bar{\varepsilon}\right]$, note that $\left|\lambda_{i}(x)-\lambda_{i}(y)\right| \leq$ $\left|\rho_{i}(x)-\rho_{i}(y)\right|$ implies that

$$
\left|p_{i}-\underline{\alpha}_{i}(z)\right|=\left|p_{i}-\lambda_{i}\left(\bar{\alpha}_{i}(z)\right)\right| \leq\left|p_{i}-\rho_{i}\left(\bar{\alpha}_{i}(z)\right)\right|=\left|p_{i}-\bar{\alpha}_{i}(z)\right|=\left|p_{i}-\bar{\alpha}(z)\right|<\bar{\varepsilon}
$$

Since $\underline{\alpha}(z) \geq \min \left\{\underline{\alpha}_{h}(z): h \in R_{i}\right\}, \mathrm{R} 2$ implies $z-\underline{\alpha}(z)<z-\underline{\alpha}_{i}(z)$, so that $p_{i}-\underline{\alpha}(z)<$ $p_{i}-\underline{\alpha}_{i}(z)$. Therefore, $\underline{\alpha}(z) \in\left[p_{i}-\bar{\varepsilon}, p_{i}+\bar{\varepsilon}\right]$ when $p_{i}-\underline{\alpha}(z) \geq 0$; and since $p_{i}-\underline{\alpha}(z)<0$ implies that $\underline{\alpha}(z) \in\left(p_{i}, z\right)$, then $\underline{\alpha}(z) \in\left[p_{i}-\bar{\varepsilon}, p_{i}+\bar{\varepsilon}\right]$ as well. To see that $\lambda_{i}(z) \in$ $\left[p_{i}-\bar{\varepsilon}, p_{i}+\bar{\varepsilon}\right]$ just note that $\left|p_{i}-\lambda_{i}(z)\right| \leq\left|p_{i}-\rho_{i}(z)\right| \leq\left|p_{i}-\bar{\alpha}_{i}(z)\right|=\left|p_{i}-\bar{\alpha}(z)\right| \leq \bar{\varepsilon}$.

Consider equilibrium proposals $x_{j+1}, x_{h+1} \in\left[p_{i}-\bar{\varepsilon}, p_{i}+\bar{\varepsilon}\right]$ such that for $j \in L_{i} \cup$ $\{i\}$ and $h \in R_{i}, x_{j}=\underline{\alpha}\left(x_{j+1}\right)$ and $x_{h}=\bar{\alpha}\left(x_{h+1}\right) \in\left[p_{i}-\bar{\varepsilon}, p_{i}+\bar{\varepsilon}\right]$. By the previous observation $\underline{\alpha}\left(x_{h+1}\right), \lambda_{i}\left(x_{h+1}\right) \in\left[p_{i}-\bar{\varepsilon}, p_{i}+\bar{\varepsilon}\right]$ as well. Using (2), yields

$$
\begin{aligned}
x_{j+1}-\underline{\alpha}\left(x_{j+1}\right) & <\lambda_{i}\left(x_{h+1}\right)-\underline{\alpha}_{i}\left(\lambda_{i}\left(x_{h+1}\right)\right)=\lambda_{i}\left(x_{h+1}\right)-\lambda_{i}\left(\underline{\alpha}_{i}\left(x_{h+1}\right)\right) \leq \\
& \leq\left|\rho_{i}\left(x_{h+1}\right)-\rho_{i}\left(\underline{\alpha}_{i}\left(x_{h+1}\right)\right)\right|=\rho_{i}\left(\underline{\alpha}_{i}\left(x_{h+1}\right)\right)-\rho_{i}\left(x_{h+1}\right)= \\
& =\bar{\alpha}_{i}\left(x_{h+1}\right)-\rho_{i}\left(x_{h+1}\right) \leq \bar{\alpha}_{i}\left(x_{h+1}\right)-x_{h+1}=\bar{\alpha}\left(x_{h+1}\right)-x_{h+1} .
\end{aligned}
$$

Hence, for any $j \in L_{i} \cup\{i\}$ and any $h \in R_{i}, a_{j}=x_{j+1}-\underline{\alpha}\left(x_{j+1}\right)<\bar{\alpha}\left(x_{h+1}\right)-x_{h+1}=b_{h}$. Since $q>n / 2$ implies that $\# R_{i} \geq \#\left(L_{i} \cup\{i\}\right)$, we conclude that

$$
\sum_{j \in L_{i} \cup\{i\}} a_{j}=\sum_{j \in L_{i} \cup\{i\}}\left(x_{j+1}-\underline{\alpha}\left(x_{j+1}\right)\right)<\sum_{h \in R_{i}}\left(\bar{\alpha}\left(x_{h+1}\right)-x_{h+1}\right)=\sum_{h \in R_{i}} b_{h}
$$

But this contradicts the hypothesis that $\mathbf{x}$ is an SSPE, since, by definition at an SSPE $\sum_{j \in L_{i} \cup\{i\}} a_{j}=\sum_{h \in R_{i}} b_{h}$

Under the assumption that $x_{i}=\bar{\alpha}\left(x_{i+1}\right)$ the symmetric argument applies, taking into account that $\#\left(R_{i} \cup\{i\}\right) \geq \# L_{i}$. 
STEP 4. Claim: There exists $\delta_{3} \in\left[\delta_{2}, 1\right)$ such that $\forall \delta \geq \delta_{3}$, it is impossible to sustain two SSPE $\mathbf{x}$ and $\mathbf{y}$ with outcomes $x<y$, and $x_{j}, y_{j} \in[0, \bar{\varepsilon}]$ for all $j \in I$. And similarly for $x_{j}, y_{j} \in[1-\bar{\varepsilon}, 1]$.

Assume that the peak identified in Step 1 is $p_{i}=p^{1}=0$. Then $q=n, L_{i}=\emptyset$, and $0=\underline{\alpha}_{i}(z) \leq \underline{\alpha}_{h}(z)$ for all $h \in R_{i}$. Since $\# R_{i} \geq n-q+1$, by Lemma $21, \underline{\alpha}$ is a contraction in $[0, \bar{\varepsilon}]$.

Moreover, there exists $\delta_{3} \in\left[\delta_{2}, 1\right)$ such that for all $\delta>\delta_{3}$ if $z \in[0, \bar{\varepsilon}]$ with $\bar{\varepsilon}<D / 2$ then $\bar{\alpha}_{h}(z) \geq \bar{\alpha}_{i}(z)$ for all $h \in R_{i}$. To see this, take $h \in R_{i}$, and note that singlepeakedness implies $\bar{\alpha}_{h}(z) \geq p_{h}$ for all $\delta \in(0,1)$. There exists $\widetilde{\delta} \in(0,1)$ (that guarantees that $r_{i}^{-1}\left(\delta u_{i}(z)\right)$ is well defined for all $\left.\delta \geq \widetilde{\delta}\right)$, such that for all $\delta \geq \widetilde{\delta}$, by definition

$$
\begin{gathered}
\bar{\alpha}_{i}(z)=r_{i}^{-1}\left(\delta u_{i}((z))\right), \\
r_{i}^{-1}\left(u_{i}(z)\right)=z .
\end{gathered}
$$

For $z \in[0, \bar{\varepsilon}]$ fixed and $\delta \geq \widetilde{\delta}$, consider the difference $r_{i}^{-1}\left(\delta u_{i}(z)\right)-r_{i}^{-1}\left(u_{i}(z)\right)$ as a the function of $\delta$; this function is continuous and has value zero at $\delta=1$. Hence, there exists $\delta_{3} \geq \max \left\{\delta_{2}, \widetilde{\delta}\right\}$ such that

$$
r_{i}^{-1}\left(\delta u_{i}(z)\right)-r_{i}^{-1}\left(u_{i}(z)\right)=\bar{\alpha}_{i}(z)-z<\bar{\varepsilon} \text { for all } \delta>\delta_{3}
$$

And, since $z \leq \bar{\varepsilon}$ and $\bar{\varepsilon}<D / 2$,

$$
\bar{\alpha}_{i}(z) \leq 2 \bar{\varepsilon}<D \leq p_{h} \leq \bar{\alpha}_{h}(z) \text { for all } \delta>\delta_{3} \text { and all } h \in R_{i}
$$


Therefore, since $\#\{i\} \geq n-q+1$, by Lemma $21, \bar{\alpha}$ is a contraction in $[0, \bar{\varepsilon}]$. Thus, for $\delta>\delta_{3}$, by Lemma 16 , the best responses are contractions in $[0, \bar{\varepsilon}]$, so that this interval admits at most one SSPE outcome.

A similar argument covers the case $p_{i}=p^{n}=1 . \square$

Taking $\widehat{\delta}=\delta_{3}$, Steps 1 to 4 imply that for $\delta \geq \widehat{\delta}$ there is a unique SSPE.

Lemma 14 Assume that the utility of player $i$ satisfies $M C D$ and symmetry, $u_{i}(x)=$ $f_{i}\left(\left|x-p_{i}\right|\right)$. Then $\underline{\alpha}_{i}$ and $\bar{\alpha}_{i}$ are contractions; that is for all $x, y \in[0,1]$ with $x \neq y$,

$$
\left|\underline{\alpha}_{i}(x)-\underline{\alpha}_{i}(y)\right|<|x-y| \text { and }\left|\bar{\alpha}_{i}(x)-\bar{\alpha}_{i}(y)\right|<|x-y| .
$$

Proof. We prove that $\left|\underline{\alpha}_{i}(x)-\underline{\alpha}_{i}(y)\right|<|x-y|$. A similar argument establishes that $\left|\bar{\alpha}_{i}(x)-\bar{\alpha}_{i}(y)\right|<|x-y|$.

Depending on the position of $x$ and $y$ relative to $p_{i}$ we distinguish three cases: (i) If $x<y \leq p_{i}$, then $y-\underline{\alpha}_{i}(y)>x-\underline{\alpha}_{i}(x) \geq 0$ follows from MCD. Moreover, singlepeakedness implies $\underline{\alpha}_{i}(y) \geq \underline{\alpha}_{i}(x)$. Therefore, $\left|\underline{\alpha}_{i}(y)-\underline{\alpha}_{i}(x)\right|<|y-x|$. (ii) If $x<$ $p_{i}<y$, by symmetry $\left|x-\lambda_{i}(y)\right|<|x-y|$. Moreover, by definition $\underline{\alpha}_{i}(y)=\underline{\alpha}_{i}\left(\lambda_{i}(y)\right)$ and by (i), $\left|\underline{\alpha}_{i}(x)-\underline{\alpha}_{i}\left(\lambda_{i}(y)\right)\right|<\left|x-\lambda_{i}(y)\right|$ (note that $\underline{\alpha}_{i}(y)=\underline{\alpha}_{i}\left(\lambda_{i}(y)\right.$ ) holds even though $\left.u_{i}(y)>u_{i}\left(\lambda_{i}(y)\right)\right)$. Hence, $\left|\underline{\alpha}_{i}(x)-\underline{\alpha}_{i}(y)\right|=\left|\underline{\alpha}_{i}(x)-\underline{\alpha}_{i}\left(\lambda_{i}(y)\right)\right|<\left|x-\lambda_{i}(y)\right|<$ $|x-y|$. (iii) If $p_{i} \leq x<y$, consider $\lambda_{i}(y)$ and $\lambda_{i}(x)$ and notice that symmetry implies that $\left|\lambda_{i}(x)-\lambda_{i}(y)\right| \leq|x-y|$, (possibly with strict inequality when $\lambda_{i}(y)=0$ ); then using (i), $\underline{\alpha}_{i}(x)=\underline{\alpha}_{i}\left(\lambda_{i}(x)\right)$, and $\underline{\alpha}_{i}(y)=\underline{\alpha}_{i}\left(\lambda_{i}(y)\right)$ we obtain $\left|\underline{\alpha}_{i}(x)-\underline{\alpha}_{i}(y)\right|=$ $\left|\underline{\alpha}_{i}\left(\lambda_{i}(x)\right)-\underline{\alpha}_{i}\left(\lambda_{i}(y)\right)\right|<\left|\lambda_{i}(x)-\lambda_{i}(y)\right| \leq|x-y|$. 
Lemma 15 If $\underline{\alpha}_{i}$ is a contraction for all $i \in I$, then $\underline{\alpha}$ is a contraction; and similarly for $\bar{\alpha}_{i}$ and $\bar{\alpha}$.

Proof. Consider any $x, y \in[0,1]$ and assume w.l.o.g. $x<y$. Since the functions $\underline{\alpha}_{i}$ are continuous for all players, there is a finite collection of alternatives $z_{k} \in[x, y]$, $x=z_{0}<z_{1}<\ldots<z_{K+1}=y$, at which the pivotal player(s) determining $\underline{\alpha}$ change(s). That is, there exists $h:\{0,1, \ldots, K\} \rightarrow I$, such that $\underline{\alpha}\left(z_{k}\right)=\underline{\alpha}_{h(k-1)}\left(z_{k}\right)=\underline{\alpha}_{h(k)}\left(z_{k}\right)$, and for $\varepsilon>0$ and sufficiently small, $\underline{\alpha}\left(z_{k}-\varepsilon\right)=\underline{\alpha}_{h(k-1)}\left(z_{k}-\varepsilon\right)$ and $\underline{\alpha}\left(z_{k}+\varepsilon\right)=\underline{\alpha}_{h(k)}\left(z_{k}+\varepsilon\right)$, where $h(k-1)$ and $h(k)$ denote the player(s) that stop and begin being pivotal at $z_{k}$ (as we move from $x$ to $y$ ). That is, the function $\underline{\alpha}$ restricted to the interval $[x, y]$ is given by

$$
\underline{\alpha}(z)=\left\{\begin{array}{cc}
\underline{\alpha}_{h(0)}(z) & \text { if } x=z_{0} \leq z \leq z_{1} \\
\underline{\alpha}_{h(1)}(z) & \text { if } z_{1} \leq z \leq z_{2} \\
\underline{\alpha}_{h(2)}(z) & \text { if } z_{2} \leq z \leq z_{3} \\
\ldots \ldots \ldots & \ldots \ldots \ldots \ldots \ldots \\
\underline{\alpha}_{h(K)}(z) & \text { if } z_{K} \leq z \leq z_{K+1}=y
\end{array}\right.
$$

where $K$ is a finite integer and $h(k) \in I$ for all $k \in\{0, \ldots K\}$.

For simplicity, write $h(0)=i$ and $h(K)=j$. Now,

$$
\begin{aligned}
|\underline{\alpha}(y)-\underline{\alpha}(x)| & =\left|\underline{\alpha}_{j}(y)-\underline{\alpha}_{j}\left(z_{K}\right)+\underline{\alpha}_{j}\left(z_{K}\right)-\underline{\alpha}_{i}(x)\right|= \\
& =\left|\underline{\alpha}_{j}(y)-\underline{\alpha}_{j}\left(z_{K}\right)+\underline{\alpha}_{h(K-1)}\left(z_{K}\right)-\underline{\alpha}_{i}(x)\right| \leq \\
& \leq\left|\underline{\alpha}_{j}(y)-\underline{\alpha}_{j}\left(z_{K}\right)\right|+\left|\underline{\alpha}_{h(K-1)}\left(z_{K}\right)-\underline{\alpha}_{i}(x)\right| .
\end{aligned}
$$


Iterating the triangle inequality and using Lemma 14, $\left|\underline{\alpha}_{k}(z)-\underline{\alpha}_{k}\left(z^{\prime}\right)\right|<\left|z-z^{\prime}\right|$ for any $z, z^{\prime} \in[0,1]$ and any $k \in I$, we obtain

$$
\begin{aligned}
|\underline{\alpha}(y)-\underline{\alpha}(x)| & \leq\left|\underline{\alpha}_{j}(y)-\underline{\alpha}_{j}\left(z_{K}\right)\right|+\sum_{k=1}^{K-1}\left|\underline{\alpha}_{h(k)}\left(z_{k+1}\right)-\underline{\alpha}_{h(k)}\left(z_{k}\right)\right|+\left|\underline{\alpha}_{i}\left(z_{1}\right)-\underline{\alpha}_{i}(x)\right|< \\
& <\left|y-z_{K}\right|+\sum_{k=1}^{K-1}\left|z_{k+1}-z_{k}\right|+\left|z_{1}-x\right|=|y-x| .
\end{aligned}
$$

A similar argument proves that $\bar{\alpha}$ is a contraction whenever $\bar{\alpha}_{i}$ is a contraction for all $i \in I$.

Lemma 16 If $\underline{\alpha}$ and $\bar{\alpha}$ are contractions in an interval $[a, b] \subset[0,1]$, then $\chi_{i}$ is also a contraction in $[a, b]$ for all $i \in I$.

Proof. Recall that for all $i \in I \chi_{i}(x) \in[\underline{\alpha}(x), \bar{\alpha}(x)]$ and that $\chi_{i}(x) \in(\underline{\alpha}(x), \bar{\alpha}(x))$ implies $\chi_{i}(x)=p_{i}$. Consider any $x, y \in[a, b], x \neq y$, and assume w.l.o.g. that $\underline{\alpha}(x) \leq$ $\underline{\alpha}(y) .13$

Next, consider in turn each possible scenario.

1. $[\underline{\alpha}(x), \bar{\alpha}(x)] \cap[\underline{\alpha}(y), \bar{\alpha}(y)]=[c, d] \neq \emptyset$, where $c=\underline{\alpha}(y)$ and $d=\min \{\bar{\alpha}(x), \bar{\alpha}(y)\}$.

(a) If $p_{i} \in[c, d]$ then $\chi_{i}(x)=\chi_{i}(y)=p_{i}$.

(b) If $p_{i}<\underline{\alpha}(x)$ then $\chi_{i}(x)=\underline{\alpha}(x)$ and $\chi_{i}(y)=\underline{\alpha}(y)$.

(c) If $\underline{\alpha}(x) \leq p_{i}<a$, then $\chi_{i}(x)=p_{i}$ and $\chi_{i}(y)=\underline{\alpha}(y)$. In this case, $0<$

$$
\chi_{i}(y)-\chi_{i}(x)=\underline{\alpha}(y)-p_{i}<\underline{\alpha}(y)-\underline{\alpha}(x) .
$$

\footnotetext{
${ }^{13}$ Note that we can have either $\bar{\alpha}(x) \leq \bar{\alpha}(y)$ or $\bar{\alpha}(x)>\bar{\alpha}(y)$.
} 
(d) If $d<p_{i} \leq \max \{\bar{\alpha}(x), \bar{\alpha}(y)\}$ then either $\chi_{i}(x)=p_{i}$ and $\chi_{i}(y)=\bar{\alpha}(y)$ when $\bar{\alpha}(x)=\max \{\bar{\alpha}(x), \bar{\alpha}(y)\}$ or $\chi_{i}(x)=\bar{\alpha}(x)$ and $\chi_{i}(y)=p_{i}$. In the first case, $0<\chi_{i}(x)-\chi_{i}(y)=p_{i}-\bar{\alpha}(y)<\bar{\alpha}(x)-\bar{\alpha}(y)$. In the second case, $0<$ $\chi_{i}(y)-\chi_{i}(x)=p_{i}-\bar{\alpha}(x)<\bar{\alpha}(y)-\bar{\alpha}(x)$.

(e) If $p_{i}>\max \{\bar{\alpha}(x), \bar{\alpha}(y)\}$ then $\chi_{i}(x)=\bar{\alpha}(x)$ and $\chi_{i}(y)=\bar{\alpha}(y)$.

2. $[\underline{\alpha}(x), \bar{\alpha}(x)] \cap[\underline{\alpha}(y), \bar{\alpha}(y)]=\emptyset$.

(a) If $p_{i}<\underline{\alpha}(x)$ then $\chi_{i}(x)=\underline{\alpha}(x)$ and $\chi_{i}(y)=\underline{\alpha}(y)$.

(b) If $\underline{\alpha}(x) \leq p_{i} \leq \bar{\alpha}(x)$ then $\chi_{i}(x)=p_{i}$ and $\chi_{i}(y)=\underline{\alpha}(y)$. In this case, $0<$ $\chi_{i}(y)-\chi_{i}(x)=\underline{\alpha}(y)-p_{i} \leq \underline{\alpha}(y)-\underline{\alpha}(x)$.

(c) If $\bar{\alpha}(x)<p_{i}<\underline{\alpha}(y)$ then $\chi_{i}(x)=\bar{\alpha}(x), \chi_{i}(y)=\underline{\alpha}(y)$. Since $\underline{\alpha}(x)<\bar{\alpha}(x)<$ $\underline{\alpha}(y), 0<\chi_{i}(y)-\chi_{i}(x)=\underline{\alpha}(y)-\bar{\alpha}(x)<\underline{\alpha}(y)-\underline{\alpha}(x)$.

(d) If $\underline{\alpha}(y) \leq p_{i} \leq \bar{\alpha}(y)$ then $\chi_{i}(x)=\bar{\alpha}(x)$ and $\chi_{i}(y)=p_{i}$. Now, $0<\chi_{i}(y)-$ $\chi_{i}(x)=p_{i}-\bar{\alpha}(x)<\bar{\alpha}(y)-\bar{\alpha}(x)$.

(e) If $p_{i}>\bar{\alpha}(y)$ then $\chi_{i}(x)=\bar{\alpha}(x)$ and $\chi_{i}(y)=\bar{\alpha}(y)$.

In case 1 (a) it is immediate that $\left|\chi_{i}(y)-\chi_{i}(x)\right|<|y-x|$. For the remaining scenarios, if $\underline{\alpha}$ and $\bar{\alpha}$ are contractions, then the inequality follows. Hence, if $\underline{\alpha}$ and $\bar{\alpha}$ are contractions then $\chi_{i}(\cdot)$ is a contraction for all $i \in I$.

Lemma 17 For each player $i \in I$ and any $\varepsilon>0$, there exists $\eta>0$ and $\delta_{\varepsilon} \in(0,1)$ such that for $\delta>\delta_{\varepsilon}$

$$
\text { if } x \in\left[p^{n-q+1}-\eta, p^{q}+\eta\right] \cap[0,1] \text { then }\left|\chi_{i}(x)-x\right|<\varepsilon
$$


Proof. Denote by $L=\left\{i \in I: p_{i} \leq p^{n-q+1}\right\}$ and by $R=\left\{i \in I: p_{i} \geq p^{q}\right\}$, and note the following two facts:

(a) Every best response proposal $\chi_{i}(x)$ must be acceptable at least to a player $j \in L$, and to a player $k \in R$. I.e., $\underline{\alpha}(x) \geq \min \left\{\underline{\alpha}_{k}(x): k \in R\right\}$ and $\bar{\alpha}(x) \leq \max \left\{\bar{\alpha}_{j}(x): j \in L\right\}$.

(b) (i) If $x \leq p_{i}$ then for each $\varepsilon>0$ there is a $\delta_{i \varepsilon} \in(0,1)$ such that $x-\underline{\alpha}_{i}(x)<\varepsilon$ for all $\delta \geq \delta_{i \varepsilon}$; and symmetrically (ii) if $x \geq p_{i}$ then for each $\varepsilon>0$ there is a $\delta_{i \varepsilon} \in(0,1)$ such that $\bar{\alpha}_{i}(x)-x<\varepsilon$ for all $\delta \geq \delta_{i \varepsilon}$. (This claim is immediate from the definitions of $\underline{\alpha}_{i}$ and $\bar{\alpha}_{i}$ and the continuity of $u_{i}$ ).

Recall that $\chi_{i}(x) \in[\underline{\alpha}(x), \bar{\alpha}(x)]$. Hence it suffices to show that for all $\varepsilon>0$ there exist $\eta>0$ and $\delta_{\varepsilon} \in(0,1)$ such that, for $x \in\left[p^{n-q+1}-\eta, p^{q}+\eta\right] \cap[0,1]$ and $\delta \geq \delta_{\varepsilon}$, $x-\underline{\alpha}(x)<\varepsilon$ and $\bar{\alpha}(x)-x<\varepsilon$. We establish this claim next.

Case 1: Consider $p^{n-q+1} \leq x \leq p^{q}$. By (a), $\chi_{i}(x)$ must be approved by some $j \in L$ and some $k \in R$. By (b)(i) for any $\varepsilon>0$ and for any $k \in R$ there is a $\delta_{k \varepsilon} \in(0,1)$ such that $x-\underline{\alpha}_{k}(x)<\varepsilon$ for all $\delta \geq \delta_{k \varepsilon}$. Taking $\delta_{R}=\max \left\{\delta_{k \varepsilon}: k \in R\right\}$, since $\underline{\alpha}(x) \geq$ $\min \left\{\underline{\alpha}_{k}(x): k \in R\right\}$, it follows that $x-\underline{\alpha}(x)<\varepsilon$ for all $\delta \geq \delta_{R}$. Similarly, by (b)(ii) we conclude that there exists $\delta_{L}$ such that $\bar{\alpha}(x)-x<\varepsilon$ for $\delta \geq \delta_{L}$. Thus, for each $\varepsilon>0$, the claim follows for $\delta \geq \delta_{\varepsilon}=\max \left\{\delta_{L}, \delta_{R}\right\}$.

Case 2: Consider $x<p^{n-q+1}$. Again, by (a) $\chi_{i}(x)$ must be approved by some $k \in R$. Using (b)(i) it is immediate that for any $\varepsilon>0$ and for any $k \in R$, there is a $\delta_{k \varepsilon} \in(0,1)$ such that $x-\underline{\alpha}_{k}(x)<\varepsilon$ for all $\delta \geq \delta_{k \varepsilon}$; taking $\delta_{R}=\max \left\{\delta_{k \varepsilon}: k \in R\right\}$ it follows that 
$0<x-\underline{\alpha}(x)<\varepsilon$ for all $\delta \geq \delta_{R}$

Now consider $\bar{\alpha}$. For any $\varepsilon>0$, we can choose $\eta_{1}>0$ arbitrarily such that (by continuity) if $x \in\left[p^{n-q+1}-\eta_{1}, p^{n-q+1}\right] \cap[0,1]$ then there exist $\phi \geq 0$ and $\varepsilon^{\prime}>0$ satisfying $\varepsilon^{\prime}+\phi<\varepsilon$ and $\rho_{i}(x)-x \leq \phi$ for players $i \in I$ with $p_{i}=p^{n-q+1}$. Morover, for players with $p_{j}<p^{n-q+1}$ if $x \in\left[p^{n-q+1}-\eta_{2}, p^{n-q+1}\right] \cap[0,1]$, where $\eta_{2}=\min \left\{p^{n-q+1}-p_{j}: p_{j}<p^{n-q+1}\right\}$, then $\rho_{j}(x)=x$.

Choose $\eta \leq \min \left\{\eta_{1}, \eta_{2}\right\}$ and consider $x \in\left[p^{n-q+1}-\eta, p^{n-q+1}\right] \cap[0,1]$. Since $\rho_{j}(x) \geq p_{j}$ for all $j \in L$, using (b)(ii), we know that there exists $\delta_{j \varepsilon^{\prime}} \in(0,1)$ such that $\bar{\alpha}_{j}\left(\rho_{j}(x)\right)-$ $\rho_{j}(x)<\varepsilon^{\prime}$ when $\delta \geq \delta_{j \varepsilon^{\prime}}$. Then, choosing $\delta_{L}=\max \left\{\delta_{j \varepsilon^{\prime}}: j \in L\right\}$ and noting that by definition $\bar{\alpha}_{j}\left(\rho_{j}(x)\right)=\bar{\alpha}_{j}(x)$, it follows that for any $j \in L$,

$$
0<\bar{\alpha}_{j}(x)-x=\left(\rho_{j}(x)-x\right)+\left(\bar{\alpha}_{j}(x)-\rho_{j}(x)\right) \leq \phi+\varepsilon^{\prime}<\varepsilon \text { when } \delta \geq \delta_{L},
$$

where we have used $\rho_{i}(x)-x \leq \phi$ for all $i$ with $p_{i}=p^{n-q+1}$ and $\rho_{j}(x)-x=0$ for all $j$ with $p_{j}<p^{n-q+1}$.

Therefore, since by (a) $\bar{\alpha}(x) \leq \max \left\{\bar{\alpha}_{j}(x): j \in L\right\}$, we have that for $x \in\left[p^{n-q+1}-\eta, p^{n-q+1}\right] \cap$ $[0,1]$ then $\bar{\alpha}(x)-x<\varepsilon$ for all $\delta \geq \delta_{L}$.

Hence for every $\varepsilon>0$, if $\eta \leq \min \left\{\eta_{1}, \eta_{2}\right\}, \delta \geq \delta_{\varepsilon}=\max \left\{\delta_{L}, \delta_{R}\right\}$, and $x \in$ $\left[p^{n-q+1}-\eta, p^{n-q+1}\right] \cap[0,1]$ then $x-\underline{\alpha}(x)<\varepsilon$ and $\bar{\alpha}(x)-x<\varepsilon$.

Case 3: The case $x>p^{q}$ is analogous to Case 2 .

Thus the claim is established and the proof is complete.

Lemma 18 Assume $q>n / 2$. For each $\varepsilon>0$ there is $\delta_{\varepsilon} \in(0,1)$ such that if $\delta \geq \delta_{\varepsilon}$ and 
$\mathbf{x}=\left(x_{1}, \ldots x_{i}, \ldots x_{n}\right)$ is an SSPE, then $\left|x_{i}-x_{1}\right|<\varepsilon$ for all $i \in I$.

\section{Proof.}

Fix an SSPE, $\mathbf{x}=\left(x_{1}, \ldots x_{i}, \ldots x_{n}\right)$, and keep in mind that $x_{j}=\chi_{j}\left(x_{j+1}\right)$.

Step 1: For all $j \in I$ and all $\eta_{j}>0$ there exists $\delta_{j \eta_{j}} \in(0,1)$ such that if $\delta \geq \delta_{j \eta_{j}}$ then $x_{j} \in\left[p^{n-q+1}-\eta_{j}, p^{q}+\eta_{j}\right] \cap[0,1]$.

Note that $q>n / 2$ implies the existence of a player $i$ with $p_{i} \in\left[p^{n-q+1}, p^{q}\right]$, and that for such player the best response $\chi_{i}\left(x_{i+1}\right)$ lies in $\left[p^{n-q+1}, p^{q}\right]$ regardless of the value of $x_{i+1}$. Hence, we may assume the following induction hypothesis: For player $i$, for any $\eta_{i}>0$ there exists $\delta_{i \eta_{i}} \in(0,1)$ such that if $\delta \geq \delta_{i \eta_{i}}$ then $x_{i} \in\left[p^{n-q+1}-\eta_{i}, p^{q}+\eta_{i}\right] \cap[0,1]$. Next we show that the induction hypothesis implies in turn that for any $\eta_{i-1}>0$ there exists $\delta_{i-1 \eta_{i-1}} \in(0,1)$ such that if $\delta \geq \delta_{i-1 \eta_{i-1}}$ then $x_{i-1} \in\left[p^{n-q+1}-\eta_{i-1}, p^{q}+\eta_{i-1}\right] \cap[0,1]$.

Under the Induction hypothesis (that guarantees that, for any $\eta_{i}>0, x_{i} \in\left[p^{n-q+1}-\eta_{i}, p^{q}+\eta_{i}\right] \cap$ $[0,1]$ provided that $\left.\delta \geq \delta_{i \eta_{i}}\right)$, by Lemma 17, for any $\varepsilon_{i}>0$ there exists $\delta_{i \varepsilon_{i}} \geq \delta_{i \eta_{i}}$ such that if $\delta \geq \delta_{i \varepsilon_{i}}$, then $\left|\chi_{i-1}\left(x_{i}\right)-x_{i}\right|<\varepsilon_{i}$. Hence, we can conclude that for any $\eta_{i-1}>0$ there exists $\delta_{i-1 \eta_{i-1}} \in(0,1)$ such that if $\delta \geq \delta_{i-1 \eta_{i-1}}$, then $x_{i-1} \in\left[p^{n-q+1}-\eta_{i-1}, p^{q}+\eta_{i-1}\right] \cap[0,1]$ ( just select $\varepsilon_{i}$ and $\eta_{i}$ such that $\varepsilon_{i}+\eta_{i} \leq \eta_{i-1}$ and $\delta_{i-1 \eta_{i-1}} \geq \delta_{i \varepsilon_{i}}$ ).

Step 2: For all $i \in I$ and all $\varepsilon_{i}>0$, there exists $0<\delta_{i}<1$ such that if $\delta \geq \delta_{i}$ then $\left|x_{i-1}-x_{i}\right|<\varepsilon_{i}$

By Lemma 17, for all $\varepsilon_{i}>0$, there exists $\eta_{i}>0$ and $\delta_{\varepsilon_{i}} \in(0,1)$ such that (when $\left.\delta \geq \delta_{\varepsilon_{i}}\right) x_{i} \in\left[p^{n-q+1}-\eta_{i}, p^{q}+\eta_{i}\right] \cap[0,1]$ implies that $\left|x_{i-1}-x_{i}\right|<\varepsilon_{i}$. Moreover, by 
the previous step, for any $\eta_{i}>0$ there exists $\delta_{i \eta_{i}} \in(0,1)$ such that if $\delta \geq \delta_{i \eta_{i}}, x_{i} \in$ $\left[p^{n-q+1}-\eta_{i}, p^{q}+\eta_{i}\right] \cap[0,1]$. Hence, selecting $\delta_{i}=\max \left\{\delta_{\varepsilon_{i}}, \delta_{i \eta_{i}}\right\}$ the claim follows.

Step 3: To complete the proof, we check that for each $\varepsilon$ we can select a $\delta_{\varepsilon}$ such that if $\delta \geq \delta_{\varepsilon}$ then $\left|x_{1}-x_{j}\right|<\varepsilon$ for all $j \in I$.

Simply choose $\varepsilon_{i}=\varepsilon / n$ and $\delta_{\varepsilon}=\max \left\{\delta_{i}: i \in I\right\}$ such that $\left|x_{i}-x_{i+1}\right|<\varepsilon_{i}$ when $\delta \geq \delta_{i}$. Then

$$
\begin{aligned}
\left|x_{j}-x_{1}\right| & =\left|x_{j}-\sum_{i=j+1}^{n-1} x_{i}+\sum_{i=j+1}^{n-1} x_{i}+x_{n}-x_{n}-x_{1}\right| \leq \\
& \leq \sum_{i=j}^{n-1}\left|x_{i}-x_{i+1}\right|+\left|x_{n}-x_{1}\right| \leq \sum_{i=j}^{n} \varepsilon_{i}<\varepsilon .
\end{aligned}
$$

Lemma 19 Consider a game with $q>n / 2$, and such that if $\mathbf{z}$ is an SSPE then $z_{i} \in$ $(z-\varepsilon / 2, z+\varepsilon / 2)$ for all $i \in I$, where $\varepsilon \in(0, D / 2)$ and $D=\min _{i, j \in I}\left\{\left|p_{i}-p_{j}\right|: p_{i} \neq p_{j}\right\}$. If $\mathbf{x}$ and $\mathbf{y}$ are two SSPE with $x<y$, then $|x-y|<\varepsilon$ and $(x-\varepsilon / 2, y+\varepsilon / 2) \cap\left\{p_{1}, \ldots, p_{n}\right\}=$ $p_{i}$ for some $i \in I$.

Proof. Step 1. We rule out $|x-y| \geq \varepsilon$.

By assumption the proposals that arise in $\mathbf{x}$ and $\mathbf{y}$ lie respectively in $(x-\varepsilon / 2, x+\varepsilon / 2)$ and $(y-\varepsilon / 2, y+\varepsilon / 2)$. If $|x-y| \geq \varepsilon$ then $(x-\varepsilon / 2, x+\varepsilon / 2) \cap(y-\varepsilon / 2, y+\varepsilon / 2)=\emptyset$, implying that $x_{i}<y_{i}$ for all $i \in I$. By Lemma 8, this implies that $x_{i}-x_{i-1}=x_{i}-$ $\chi_{i-1}\left(x_{i}\right)<y_{i}-\chi_{i-1}\left(y_{i}\right)=y_{i}-y_{i-1}$ for all $i \in I$, and Lemma 9 implies a contradiction.

STEP 2. $|x-y|<\varepsilon$ and $(x-\varepsilon / 2, y+\varepsilon / 2) \cap\left\{p_{1}, \ldots, p_{n}\right\}=p_{i}$. 
Assume that there are two equilibria, $\mathbf{x}$ and $\mathbf{y}$, with outcomes $x<y$, and proposals lying on an interval with no peaks, say $x_{i}, y_{i} \in\left(p^{k}, p^{k+1}\right)$ for all $i \in I$. Then it is necessarily the case that $\left(p^{k}, p^{k+1}\right) \subset\left[p^{n-q+1}, p^{q}\right]$; otherwise, for player $j$ with $p_{j} \in\left[p^{n-q+1}, p^{q}\right]$ the equilibrium proposal must be $\chi_{j}\left(x_{j+1}\right) \in\left[p^{n-q+1}, p^{q}\right] \not \subset\left(p^{k}, p^{k+1}\right)$. If all proposals in both equilibria lie in $\left(p^{k}, p^{k+1}\right) \subset\left[p^{n-q+1}, p^{q}\right]$, for all $i \in I$, if $x_{i}=\underline{\alpha}\left(x_{i+1}\right)$ then $y_{i}=\underline{\alpha}\left(y_{i+1}\right)$, and similarly if $x_{i}=\bar{\alpha}\left(x_{i+1}\right)$ then $y_{i}=\bar{\alpha}\left(y_{i+1}\right)$. Hence, for $\mathbf{z}=\mathbf{x}, \mathbf{y}$, if player $i$ proposes $z_{i}=\underline{\alpha}\left(z_{i+1}\right)$ then $\underline{\alpha}\left(z_{i+1}\right)=\underline{\alpha}^{R, q-k}\left(z_{i+1}\right)$ where $R=\left\{i \in I: p_{i} \geq p^{k+1}\right\}$ and if $j$ proposes $z_{j}=\bar{\alpha}\left(z_{j+1}\right)$ then $\bar{\alpha}\left(z_{j+1}\right)=\bar{\alpha}^{L, q-(n-k)}\left(z_{j+1}\right)$ where $L=\left\{i \in I: p_{i} \leq p^{k}\right\}$. By singlepeakedness, $x_{i+1}<y_{i+1}$ implies $\underline{\alpha}_{k}\left(x_{i+1}\right) \leq \underline{\alpha}_{k}\left(y_{i+1}\right)$ for all $k \in R$ and $\bar{\alpha}_{k}\left(x_{i+1}\right) \leq$ $\bar{\alpha}_{k}\left(y_{i+1}\right)$ for all $k \in L$, so that $\underline{\alpha}\left(x_{i+1}\right) \leq \underline{\alpha}\left(y_{i+1}\right)$ and $\bar{\alpha}\left(x_{j+1}\right) \leq \bar{\alpha}\left(y_{j+1}\right)$, where $i$ and $j$ denote players proposing $z_{i}=\underline{\alpha}\left(z_{i+1}\right)$ and $z_{j}=\bar{\alpha}\left(z_{j+1}\right)$ respectively, for $z=x, y$. Hence, if $x_{i+1}<y_{i+1}$ then $\chi_{i}\left(x_{i+1}\right)=x_{i}<y_{i}=\chi_{i}\left(y_{i+1}\right)$. Since $x_{1}=x<y=y_{1}$, then $x_{i}<y_{i}$ for all $i \in I$. Therefore, by Lemma $8, x_{i+1}-x_{i}<y_{i+1}-y_{i}$ for any $i \in I$; and by Lemma 9 we derive a contradiction.

Hence $|x-y|<\varepsilon$ and $(x-\varepsilon / 2, y+\varepsilon / 2) \cap\left\{p_{1}, \ldots, p_{n}\right\} \neq \emptyset$. Moreover, since $\varepsilon<D / 2$, $(x-\varepsilon / 2, y+\varepsilon / 2) \cap\left\{p_{1}, \ldots, p_{n}\right\}$ must be singleton, so that the result follows.

Lemma 20 For every profile of continuous and single-peaked utility functions there exist $\varepsilon \in(0,1)$, such that the following holds for all $i \in I$ :

SIGN: Either $\left|\lambda_{i}(x)-\lambda_{i}(y)\right| \leq\left|\rho_{i}(x)-\rho_{i}(y)\right|$ for all $x, y \in[p-\varepsilon, p+\varepsilon]$, or the reverse weak inequality holds throughout this interval. 
Proof. Consider any player with peak $p_{i}=p$ and utility $u_{i}=u$. The claim is obvious when $p \in\{0,1\}$, since either $\lambda(x)=\lambda(y)=0$ or $\rho(x)=\rho(y)=1$ for all $x, y \in$ $[p-\varepsilon, p+\varepsilon] \cap[0,1]$, for any $\varepsilon>0$.

Assume $p \in(0,1)$, let $\underline{u}=\max \{u(0), u(1)\}<u(p)$ and consider $l^{-1}:[\underline{u}, u(p)] \rightarrow[0,1]$ and $r^{-1}:[\underline{u}, u(p)] \rightarrow[0,1]$. These functions are increasing and decreasing respectively, continuous and (by definition) $l^{-1}(u(p))=r^{-1}(u(p))=p$. Define function $f:[\underline{u}, u(p)] \rightarrow[0,1]$ as $f(u)=\left[r^{-1}(u)-p\right]-\left[p-l^{-1}(u)\right]$. Since $f$ is continuous and $f(u(p))=0$, there exists $\bar{u} \in[\underline{u}, u(p)]$ such that for all $u \in[\bar{u}, u(p)]$ either (i) $f$ is non-increasing and $f(u) \geq 0$, or (ii) $f$ is non-decreasing and $f(u) \leq 0$.

Take an $\varepsilon>0$ such that for all $z \in[p-\varepsilon, p+\varepsilon]$, both $u(\lambda(z)) \geq \bar{u}$ and $u(\rho(z)) \geq \bar{u}$. We will show next that either $|\lambda(x)-\lambda(y)| \leq|\rho(x)-\rho(y)|$ for all $x, y \in[p-\varepsilon, p+\varepsilon]$, or the reverse weak inequality holds throughout this interval.

Assume (i), consider any $x, y \in[p-\varepsilon, p+\varepsilon]$, and assume w.l.o.g. that $\rho(x)<\rho(y)$, which implies (by single-peakedness) $\lambda(x)>\lambda(y)$ (note that $\rho(x)=\rho(y)$ implies $\lambda(x)=$ $\lambda(y)$ and either weak inequality holds trivially). Since $u(\rho(x))>u(\rho(y))$, by (i) we have that $f(u(\rho(x)))-f(u(\rho(y))) \leq 0$; that is

$$
r^{-1}(u(\rho(x)))+l^{-1}(u(\rho(x)))-r^{-1}(u(\rho(y)))-l^{-1}(u(\rho(y))) \leq 0
$$

Since by definition, $r^{-1}(u(\rho(z)))=\rho(z)$ and $l^{-1}(u(\rho(z)))=\lambda(z)$ for $z=x$, y, we get $\rho(x)+\lambda(x)-\rho(y)-\lambda(y) \leq 0$, or equivalently,

$$
\lambda(x)-\lambda(y) \leq \rho(y)-\rho(x) .
$$


Therefore, since $\rho(x)<\rho(y)$ and $\lambda(x)>\lambda(y)$, we conclude that

$$
|\lambda(x)-\lambda(y)| \leq|\rho(x)-\rho(y)| \text { for all } x, y \in[p-\varepsilon, p+\varepsilon]
$$

Similar arguments apply to case (ii). Then $f$ non-decreasing and $f(z) \leq 0$ for all $z \in$ $[\bar{u}, u(p)]$, implies that $|\lambda(x)-\lambda(y)| \geq|\rho(x)-\rho(y)|$ for all $x, y \in[p-\varepsilon, p+\varepsilon]$.

Lemma 21 Consider a profile of $M C D$ utilities. Let $[a, b] \subset[0,1]$ and $Q \subset I$,\#Q $\geq$ $n-q+1$.

(i) If $p_{j} \geq b$ for all $j \in Q$ and $\underline{\alpha}_{j}(z) \geq \underline{\alpha}_{k}(z)$ for all $z \in[a, b]$ and all $k \in I \backslash Q$, then $\underline{\alpha}$ is a contraction in $[a, b]$.

(ii) If $p_{j} \leq a$ for all $j \in Q$ and $\bar{\alpha}_{j}(z) \leq \bar{\alpha}_{k}(z)$ for all $z \in[a, b]$ and all $k \in I \backslash Q$, then $\bar{\alpha}$ is a contraction in $[a, b]$.

Proof. We prove statement (i), the proof of (ii) is analogous. Let $s=q-\#(I \backslash Q)$. Since $\underline{\alpha}(z)=\underline{\alpha}^{I, q}(x)$ and, for any $j \in Q, \underline{\alpha}_{j}(z) \geq \underline{\alpha}_{k}(z)$ for all $k \in I \backslash Q$, then $\underline{\alpha}(z)=\underline{\alpha}^{Q, s}(x)$. Consider $z, z^{\prime} \in[a, b], z<z^{\prime}$. Since $\# Q \geq n-q+1$, there are at least $n-q+1$ players $j \in Q$ such that $\underline{\alpha}_{j}\left(z^{\prime}\right) \geq \underline{\alpha}\left(z^{\prime}\right)$. Since $\underline{\alpha}(z)$ must be acceptable at least to one of those players, say $h \in Q$, it must be that $\underline{\alpha}_{h}(z) \leq \underline{\alpha}(z)$. Thus, MCD implies $z-\underline{\alpha}(z) \leq z-\underline{\alpha}_{h}(z)<z^{\prime}-\underline{\alpha}_{h}\left(z^{\prime}\right) \leq z^{\prime}-\underline{\alpha}\left(z^{\prime}\right)$. Moreover, single-peakedness assures that $\underline{\alpha}_{j}(z) \leq \underline{\alpha}_{j}\left(z^{\prime}\right)$ for all $j \in Q$ and therefore, since $\underline{\alpha}(x)=\underline{\alpha}^{Q, s}(x)$, it must be that $\underline{\alpha}(z) \leq \underline{\alpha}\left(z^{\prime}\right)$. Thus, $\left|\underline{\alpha}\left(z^{\prime}\right)-\underline{\alpha}(z)\right|<\left|z^{\prime}-z\right|$ for all $z, z^{\prime} \in[a, b]$. 